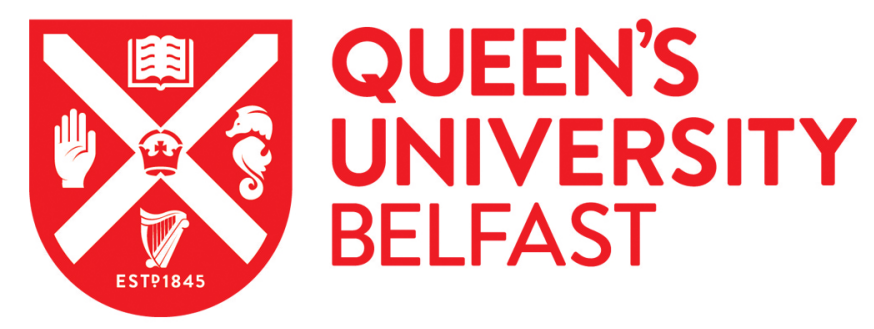

\title{
Masses and radii for the three super-Earths orbiting GJ 9827, and implications for the composition of small exoplanets
}

Rice, K., Malavolta, L., Mayo, A., Mortier, A., Buchhave, L. A., Affer, L., Vanderburg, A., Lopez-Morales, M., Poretti, E., Zeng, L., Cameron, A. C., Damasso, M., Coffinet, A., Latham, D. W., Bonomo, A. S., Bouchy, F., Charbonneau, D., Dumusque, X., Figueira, P., ... Watson, C. (2019). Masses and radii for the three super-Ëarths orbiting GJ 9827, and implications for the composition of small exoplanets. Monthly Notices of the Royal Astronomical Society, 484(3), 3731-3745. https://doi.org/10.1093/mnras/stz130

Published in:

Monthly Notices of the Royal Astronomical Society

Document Version:

Peer reviewed version

Queen's University Belfast - Research Portal:

Link to publication record in Queen's University Belfast Research Portal

Publisher rights

Copyright 2018 OUP. This work is made available online in accordance with the publisher's policies. Please refer to any applicable terms of use of the publisher.

\section{General rights}

Copyright for the publications made accessible via the Queen's University Belfast Research Portal is retained by the author(s) and / or other copyright owners and it is a condition of accessing these publications that users recognise and abide by the legal requirements associated with these rights.

Take down policy

The Research Portal is Queen's institutional repository that provides access to Queen's research output. Every effort has been made to ensure that content in the Research Portal does not infringe any person's rights, or applicable UK laws. If you discover content in the Research Portal that you believe breaches copyright or violates any law, please contact openaccess@qub.ac.uk. 


\section{Masses and radii for the three super-Earths orbiting GJ 9827, and implications for the composition of small exoplanets}
K. Rice ${ }^{1,2 \star}$, L. Malavolta ${ }^{3,4}$, A. Mayo ${ }^{5,6,7}$, A. Mortier ${ }^{8,9}$, L.A. Buchhave ${ }^{10}$, L. Affer ${ }^{11}$, A. Vanderburg ${ }^{12,13,14}$, M. Lopez-Morales ${ }^{13}$, E. Poretti ${ }^{15,16}$, L. Zeng ${ }^{17}$, A.C. Cameron ${ }^{9}$, M. Damasso ${ }^{18}$, A. Coffinet ${ }^{19}$, D. W. Latham ${ }^{13}$, A.S. Bonomo ${ }^{18}$, F. Bouchy ${ }^{19}$, D. Charbonneau ${ }^{13}$, X. Dumusque ${ }^{19}$, P. Figueira ${ }^{20,21}$, A.F. Martinez Fiorenzano ${ }^{15}$, R.D. Haywood ${ }^{13,14}$,

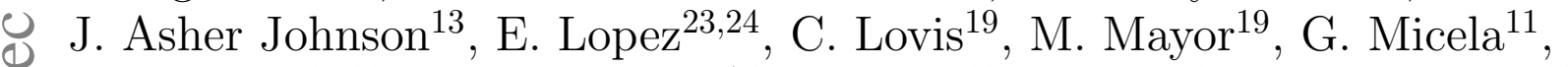
E. Molinari ${ }^{15,22}$, V. Nascimbeni ${ }^{4,3}$, C. Nava ${ }^{13}$, F. Pepe ${ }^{19}$, D. F. Phillips ${ }^{13}$, G. Piotto ${ }^{4,3}$, D. Sasselov ${ }^{13}$, D. Ségransan ${ }^{19}$, A. Sozzetti ${ }^{18}$, S. Udry ${ }^{19}$, C. Watson ${ }^{25}$

\footnotetext{
${ }^{1}$ SUPA, Institute for Astronomy, University of Edinburgh, Royal Observatory, Blackford Hill, Edinburgh, EH93HJ, UK

${ }^{2}$ Centre for Exoplanet Science, University of Edinburgh, Edinburgh, UK

${ }^{3}$ INAF - Osservatorio Astronomico di Padova, Vicolo dell'Osservatorio 5, 35122 Padova, Italy

${ }^{4}$ Dipartimento di Fisica e Astronomia "Galileo Galilei", Universita'di Padova, Vicolo dell'Osservatorio 3, 35122 Padova, Italy

${ }^{5}$ Astronomy Department, University of California, Berkeley, CA 94720, USA

${ }^{6}$ National Science Foundation Graduate Research Fellow

${ }^{7}$ Fulbright Fellow

${ }^{8}$ Astrophysics group, Cavendish Laboratory, University of Cambridge, J.J. Thomson Avenue, Cambridge CB3 OHE, UK

${ }^{9}$ Centre for Exoplanet Science, SUPA, School of Physics and Astronomy, University of St Andrews, St Andrews KY169SS, UK

${ }^{10}$ DTU Space, National Space Institute, Technical University of Denmark, Elektrovej 327, DK-2800 Lyngby, Denmark

${ }^{11}$ INAF - Osservatorio Astronomico di Palermo, Piazza del Parlamento 1, I-90134 Palermo, Italy

12 Department of Astronomy, The University of Texas at Austin, 2515 Speedway, Stop C1400, Austin, TX 78712

${ }^{13}$ Harvard-Smithsonian Center for Astrophysics, 60 Garden Street, Cambridge, MA 02138, USA

${ }^{14}$ NASA Sagan Fellow

${ }^{15}$ INAF - Fundación Galileo Galilei, Rambla José Ana Fernandez Pérez 7, 38712 Breña Baja, Spain

${ }^{16}$ INAF - Osservatorio Astronomico di Brera, Via E. Bianchi 46, 23807 Merate, Italy

${ }^{17}$ Department of Earth and Planetary Sciences, Harvard University, Cambridge, MA 02138, USA

${ }^{18}$ INAF - Osservatorio Astrofisico di Torino, Via Osservatorio 20, I-10025 Pino Torinese, Italy

${ }^{19}$ Observatoire de Genève, Université de Genève, $51 \mathrm{ch}$. des Maillettes, 1290 Sauverny, Switzerland

${ }^{20}$ European Southern Observatory, Alonso de Cordova 3107, Vitacura, Santiago, Chile

${ }^{21}$ Instituto de Astrofísica e Ciências do Espaço, Universidade do Porto, CAUP, Rua das Estrelas, 4150-762 Porto, Portugal

${ }^{22}$ INAF - Osservatorio di Cagliari, via della Scienza 5, 09047 Selargius, CA, Italy

${ }^{23}$ NASA Goddard Space Flight Center, 8800 Greenbelt Rd, Greenbelt, MD 20771, USA

${ }^{24}$ GSFC Sellers Exoplanet Environments Collaboration, NASA GSFC, Greenbelt, MD 20771

${ }^{25}$ Astrophysics Research Centre, School of Mathematics and Physics, Queen's University Belfast, Belfast BT7 1NN, UK
}

Accepted XXX. Received YYY; in original form ZZZ 


\begin{abstract}
Super-Earths belong to a class of planet not found in the Solar System, but which appear common in the Galaxy. Given that some super-Earths are rocky, while others retain substantial atmospheres, their study can provide clues as to the formation of both rocky planets and gaseous planets, and - in particular - they can help to constrain the role of photo-evaporation in sculpting the exoplanet population. GJ 9827 is a system already known to host 3 super-Earths with orbital periods of 1.2 , 3.6 and 6.2 days. Here we use new HARPS-N radial velocity measurements, together with previously published radial velocities, to better constrain the properties of the GJ 9827 planets. Our analysis can't place a strong constraint on the mass of GJ 9827 c, but does indicate that GJ 9827 b is rocky with a composition that is probably similar to that of the Earth, while GJ 9827 d almost certainly retains a volatile envelope. Therefore, GJ 9827 hosts planets on either side of the radius gap that appears to divide super-Earths into pre-dominantly rocky ones that have radii below $\sim 1.5 R_{\oplus}$, and ones that still retain a substantial atmosphere and/or volatile components, and have radii above $\sim 2 R_{\oplus}$. That the less heavily irradiated of the 3 planets still retains an atmosphere, may indicate that photoevaporation has played a key role in the evolution of the planets in this system.
\end{abstract}

Key words: Stars: individual: GJ 9827 (2MASS J23270480-0117108, EPIC 246389858, HIP 115752) - Planets and satellites: fundamental parameters - Planets and satellites: composition - Planets and satellites: general - Planets and satellites: detection - Techniques: radial velocities

\section{INTRODUCTION}

One of the most exciting recent exoplanet results is the discovery that the most common type of exoplanet, with a period less than $\sim 100$ days, is one with a radius between that of the Earth $\left(1 R_{\oplus}\right)$ and that of Neptune $\left(\sim 4 R_{\oplus}\right)$ (Howard et al. 2012; Batalha et al. 2013; Fulton et al. 2017; Fulton \& Petigura 2018). Known as super-Earths, these appear to be common in the Galaxy, but are not found in our Solar System. It also appears that the transition from being preferentially rocky/terrestrial to having a substantial gaseous atmosphere occurs within this size range (Rogers 2015). Recent studies (Fulton et al. 2017; Zeng et al. 2017; Van Eylen et al. 2018) have suggested that there is in fact a gap in the radius distribution between 1.5 and $2 R_{\oplus}$, as predicted by Owen \& Wu (2013) and Lopez \& Fortney (2013). Planets tend to have radii less than $\sim 1.5 R_{\oplus}$ and may be predominantly rocky, or they sustain a substantial gaseous envelope and have radii above $2 R_{\oplus}$.

Super-Earths are, therefore, an important population as they may provide clues as to both the formation of gas giants and the formation of rocky, terrestrial planets. In particular, they can help us to better understand the role that photo-evaporation plays in sculpting the exoplanet population. It has been suggested that super-Earths probably formed with gas envelopes that make up at least a few percent of their mass (Rogers et al. 2011; Lopez \& Fortney 2014; Wolfgang \& Lopez 2015). Those that are sufficiently strongly irradiated could then have lost their atmospheres

* Email: wkmr@roe.ac.uk via photo-evaporation (Lopez et al. 2012; Owen \& Wu 2013; Ehrenreich et al. 2015). Those that have not been sufficiently strongly irradiated retain their atmospheres. This could then explain the observed gap in the radius distribution (Owen \& Wu 2017; Fulton et al. 2017; Lopez \& Rice 2018; Van Eylen et al. 2018). There may, however, be alternative explanations for this observed radius gap, such as late giant impacts (Inamdar \& Schlichting 2015) or the atmosphere being stripped by the cooling rocky core (Ginzburg et al. 2016, 2018). This means that systems that have super-Earths on either side of this radius gap are particularly interesting.

In this paper we present an analysis of one such system, the $K 2$ target GJ 9827 (also known as K2-135, EPIC 246389858 , or HIP 115752). It is already known to host 3 super-Earths with radii between 1 and $\sim 2 R_{\oplus}$, and with orbital periods of 1.21 days, 3.65 days, and 6.21 days (Niraula et al. 2017; Rodriguez et al. 2018). Rodriguez et al. (2018) and Niraula et al. (2017) suggest that GJ 9827 b has a radius of $\sim 1.6 R_{\oplus}$, GJ $9827 \mathrm{c}$ has a radius of $\sim 1.3 R_{\oplus}$, while GJ $9827 \mathrm{~d}$ has a radius of about $2 R_{\oplus}$. This means that these planets have radii that approximately bracket the radius gap detected by Fulton et al. (2017) which, as already suggested, makes this a particularly interesting system for studying the origin of this gap. However, neither Rodriguez et al. (2018) nor Niraula et al. (2017) could independently estimate the planets masses and so used mass-radius relations (Weiss \& Marcy 2014; Chen \& Kipping 2017).

A recent radial velocity analysis (Teske et al. 2018) has, however, presented mass estimates for the GJ 9827 planets. This analysis was unable to place strong constraints on the masses of GJ $9827 \mathrm{c}$ and d, but suggests that GJ $9827 \mathrm{~b}$ has a mass of $\sim 8.2 \pm 1.53 M_{\oplus}$. With a radius of $\sim 1.64 R_{\oplus}$ (Rodriguez et al. 2018; Niraula et al. 2017), this result would make GJ 9827 b one of the densest known super-Earths. This mass and radius would suggest that GJ $9827 \mathrm{~b}$ has an 
iron core that makes up a significant fraction of its mass, and could indicate that it has undergone a mantle-stripping collision with another body of a similar mass (Marcus et al. 2010). A more recent analysis (Prieto-Arranz et al. 2018), however, suggests that the mass of GJ $9827 \mathrm{~b}$ is not as high as suggested by Teske et al. (2018) and, in fact, may have a composition similar to that of the Earth. This analysis also suggests that GJ 9827 c is also rocky, but that GJ $9827 \mathrm{~d}$ may retain a substantial, extended atmosphere.

Here we repeat the lightcurve analysis of GJ 9827 using the $K 2$ data, which we present in Section 4 . We also use the $K 2$ lightcurve to constrain the stellar activity (Section $5)$. We then carry out a radial velocity analysis using the same radial velocity data as used by Teske et al. (2018), Niraula et al. (2017) and Prieto-Arranz et al. (2018), but with an additional 41 new radial velocities from the HARPS-N spectrograph (Cosentino et al. 2012, 2014). As we will discuss in Section 7, we were able to constrain the masses of GJ 9827 b and d to better than "10\%" and about "20\%", but were not able to place a strong constraint on the mass of GJ 9827 c. We also discuss what these results imply about the typical composition of planets below the radius gap (Fulton et al. 2017), a particular science goal of the HARPS-N Collaboration.

\section{RADIAL VELOCITY OBSERVATIONS}

\subsection{HARPS-N spectroscopy}

We collected a total of 43 radial velocity (RV) spectra of GJ 9827 with the HARPS-N spectrograph $(\mathrm{R}=115000)$ installed on the 3.6-m Telescopio Nazionale Galileo (TNG) at the Observatorio de los Muchachos in La Palma, Spain (Cosentino et al. 2012, 2014). We observed GJ 9827 between August 2017 and December 2017 as part of the HARPSN Collaboration's Guaranteed Time Observations (GTO) program. Our observational strategy consisted of taking one or two observations per night, separated by 2-3 hours, for several consecutive nights in order to properly sample the RV curve of all the transiting planets.

All the observations had an exposure time of 1800s. We eliminated one observation, taken on $\mathrm{BJD}=2458048.36$, as it had an anomalously low signal-to-noise ratio (S/N) of less than 20 , and another, taken on $\mathrm{BJD}=2457991.62$, was rejected by the data reduction software because of abnormal flux correction.

GJ 9827 has a V-band magnitude of $\mathrm{V}=10.25$, so, with the exception of the two observations that were eliminated, we obtained spectra with signal-to-noise ratios in the range $\mathrm{S} / \mathrm{N}=37-121$ (average $\mathrm{S} / \mathrm{N}=70$ ), at $550 \mathrm{~nm}$ in 30 minute exposures, resulting in an average RV precision of $1.9 \mathrm{~m} \mathrm{~s}^{-1}$.

The spectra were reduced with version 3.8 of the HARPS-N Data Reduction Software (DRS), which includes corrections for color systematics introduced by variations in seeing (Cosentino et al. 2014). The radial velocities were computed using a numerical weighted mask based on the synthetic spectrum of a K5 dwarf, following the methodology outlined in Baranne et al. (1996) and Pepe et al. (2002). The HARPS-N data are presented in Table 6 and the radial velocities are shown in Figure 1. Table 6 also includes some stellar activity indicators. Specifically, the full width at half

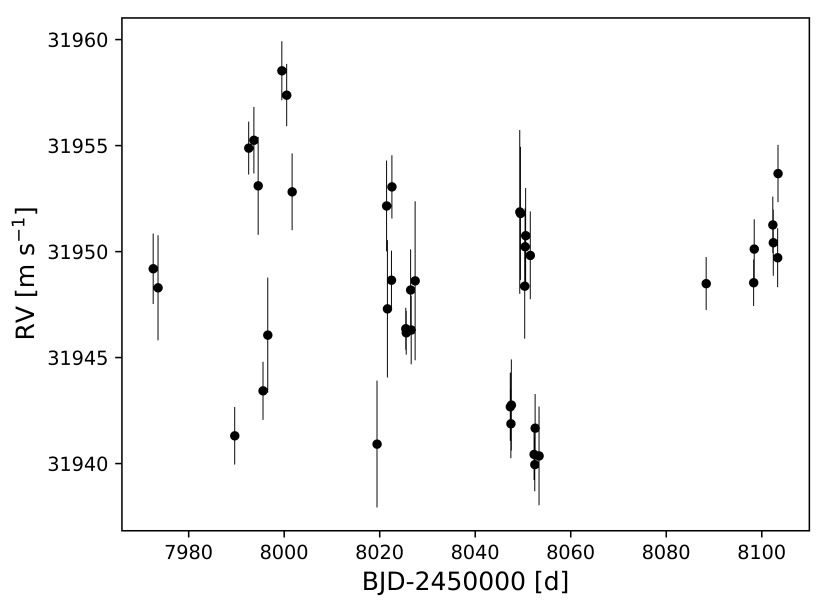

Figure 1. The HARPS-N radial velocities, first presented here, plotted against time.

maximum (FWHM) of the cross-correlation function (CCF), the line Bisector Inverse Slope (BIS), and an activity index derived from the Calcium $\mathrm{H}$ and $\mathrm{K}$ lines $\left(\mathrm{S}_{\mathrm{HK}}\right)$.

\subsection{Previously published HARPS-N and HARPS spectroscopy}

In our analysis we also use the HARPS-N and HARPS spectra first presented in Prieto-Arranz et al. (2018). This additional dataset includes 23 HARPS-N RV spectra, taken between July 2017 and December 2017, and 35 HARPS spectra taken between August 2017 and October 2017. The HARPS instrument is installed on the $3.6 \mathrm{~m}$ ESO telescope at La Silla and is very similar to the HARPS-N instrument, already discussed in Section 2.1. The HARPS and HARPS-N spectra were reduced with the same DRS as the HARPS-N spectra presented in Section 2.1. The HARPS-N RVs presented by Prieto-Arranz et al. (2018) have an average precision of 1.6 $\mathrm{m} \mathrm{s}^{-1}$ and signal-to-noise ratios in the range 33 - 95 (average $\mathrm{S} / \mathrm{N}=68$ ), while the HARPS RVs have an average precision of of $1.4 \mathrm{~m} \mathrm{~s}^{-1}$ and signal-to-noise ratios in the range $47-100$ (average $\mathrm{S} / \mathrm{N}=76$ ).

The Prieto-Arranz et al. (2018) HARPS and HARPS$\mathrm{N}$ data, including stellar activity indicators, can be found in their Tables 2 and 3, respectively. Their HARPS-N and HARPS RVs, together with the new HARPS-N RVs presented here, are shown in the top panel of Figure 2. The filled circles show the new HARPS-N RVs from this study, the open circles show the HARPS-N RVs from Prieto-Arranz et al. (2018), and the open squares show their HARPS RVs. We also correct for the RV offset, but assume that all the HARPS-N data has the same offset ${ }^{1}$, while allowing the HARPS and HARPS-N data to have different offsets. This is discussed in more detail in Section 6.

1 We verified that the two HARPS-N datasets were obtained using the same instrumental setup, and analyzed with the same version of the pipeline and using the same RV mask 


\subsection{Previously published Magellan/PFS and NOT/FIES spectroscopy}

In addition to the HARPS-N and HARPS spectra, we also include in our analysis the Magellan/PFS observations first presented by Teske et al. (2018). Thirty-six PFS observations were taken between January 2010 and August 2016, using the Planet Finder Spectrograph (PFS: Crane et al. 2006) on the Magellan II (Clay) Telescope. The resolution was $\sim 80000$ and the exposure times were between $457 \mathrm{~s}$ and 900 s. More details can be found in Teske et al. (2018), and the resulting radial velocities are shown in their Table 1.

Similarly, we also include the 7 high-resolution $(R \sim$ $67000)$ spectra taken using the FIbre-fed Échelle Spectrograph (FIES: Telting et al. 2014) on the $2.6 \mathrm{~m}$ Nordic Optical Telescope (NOT) of the Roque de los Muchachos Observatory (La Palma, Spain). More details can be found in Niraula et al. (2017), in which these observations were first presented, and the resulting radial velocities are shown in their Table 2.

The bottom panel of Figure 2 shows the PFS (blue squares) and FIES (red triangles) radial velocities, together with the new HARPS-N RVs from this study (black filled circles) and the HARPS-N and HARPS RVs presented by Prieto-Arranz et al. (2018) (black open circles and black open squares respectively). These are all corrected for offsets between the datasets based on the best RV fit for the combined datasets discussed in Section 6 .

\section{STELLAR PARAMETERS}

Taking advantage of the high $\mathrm{S} / \mathrm{N}$, high-resolution spectra obtained using HARPS-N, we re-determined the stellar parameters of GJ 9827 using the Stellar Parameter Classification pipeline (SPC; Buchhave et al. 2014).

The high $\mathrm{S} / \mathrm{N}$ needed to extract precise RVs means that these spectra are more than adequate for deriving stellar parameters. Using the spectra obtained through the HARPS-N GTO program, we ran the SPC analysis on each individual spectrum, with a prior on the surface gravity from the YY isochrone models (Spada et al. 2013). This SPC analysis yielded: $T_{\text {eff }}=4305 \pm 49 \mathrm{~K}, \log g=4.72 \pm 0.10(\mathrm{cgs}),[\mathrm{m} / \mathrm{H}]$ $=-0.50 \pm 0.08$ and $v \sin i<2 \mathrm{~km} \mathrm{~s}^{-1}$. The formal uncertainties also take into account the model uncertainties, which primarily stem from model systematics in the ATLAS Kurucz stellar models and degeneracies between the derived parameters when trying to compare observed spectra to model spectra (see e.g., Buchhave et al. 2012, 2014).

To determine the mass, $M_{\star}$, and radius, $R_{\star}$, of GJ 9827, we used the isochrones Python package (Morton 2015), which uses both the Mesa Isochrones and Stellar Tracks (MIST: Dotter 2016) and the Dartmouth Stellar Evolution Database (Dotter et al. 2008). In addition to $T_{\text {eff }}, \log g$ and $[\mathrm{m} / \mathrm{H}]$, we included as priors the AAVSO Photometric AllSky Survey $B$ and $V$ magnitudes (Henden et al. 2015), the 2MASS $J$ and $K$ magnitudes (Skrutskie et al. 2006), the WISE2,3 and 4 magnitudes (Cutri \& et al. 2014), and the Gaia parallax from Data Release 2 (Gaia Collaboration et al. 2016, 2018). We also repeat this analysis using the Hipparcos parallax (van Leeuwen 2007), to see how this impacts the resulting mass and radius estimates. We used both the
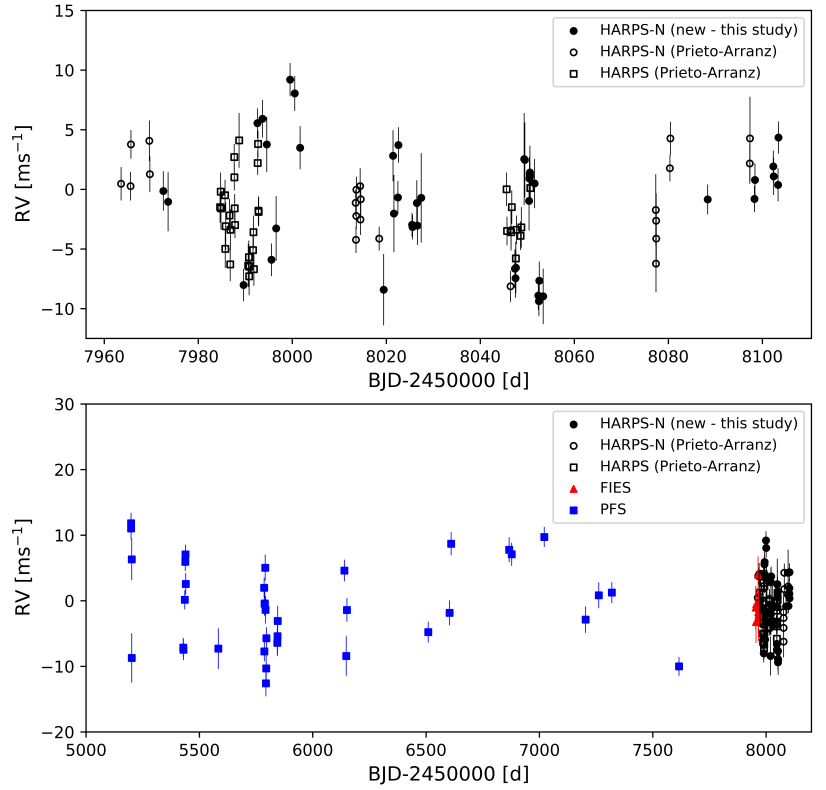

Figure 2. Top panel: The new HARPS-N RVs from this study (black filled circles) together with the HARPS-N and HARPS RVs presented by Prieto-Arranz et al. (2018) (black open circles and black open squares respectively). Bottom panel: PFS (blue squares) and FIES (red triangles) RVs, together with all the HARPS-N and HARPS RVs (black filled circles, black open circles, and black open squares), all corrected for offsets between the datasets.

MIST and Dartmouth model grids. Posterior sampling was performed using MultiNest (Feroz \& Hobson 2008; Feroz et al. 2009, 2013).

In order to investigate the systematic errors on $M_{*}$ and $R_{*}$ introduced by the spectrally-derived stellar parameters when dealing with late $\mathrm{K}$ and cooler dwarfs, we repeated the analysis using the stellar atmosphere parameters from Niraula et al. (2017), Teske et al. (2018), and Rodriguez et al. (2018). Niraula et al. (2017) and Teske et al. (2018) used SpecMatch-Emp (Yee et al. 2017), the results of which are shown in their Table 3 and Table 2 respectively. The stellar parameters used by Rodriguez et al. (2018) are shown in their Table 1 and are taken from Houdebine et al. (2016), who used principal component analysis.

The results of our analysis are shown in Figure 3. The thin lines show the results using the Hipparcos parallax as a prior, while the thick lines are the results obtained using the Gaia parallax as a prior.

We then produce final estimates for each parameter by taking the median and $15.865^{\text {th }} / 84.135^{\text {th }}$ percentiles of the posterior samplings for all of the sets of stellar parameters, and for both the analysis using the Gaia parallax, and the analysis using the Hipparcos parallax. The mass and radius obtained using the Hipparcos parallax as a prior are, $M_{\star}=0.60_{-0.02}^{+0.03} M_{\odot}$ and $R_{\star}=0.59_{-0.02}^{+0.02} R_{\odot}$, while using the Gaia parallax returns $M_{\star}=0.606_{-0.014}^{+0.020} M_{\odot}$ and 


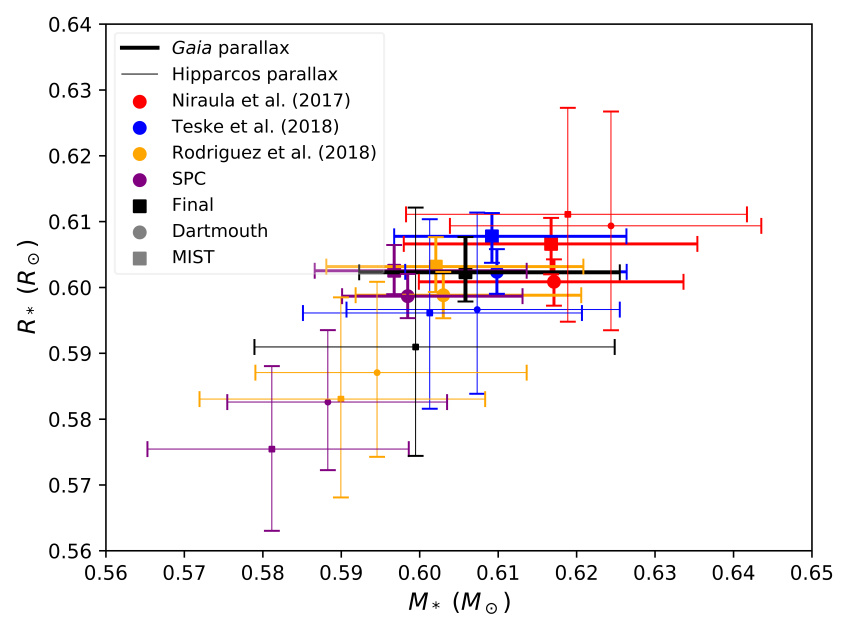

Figure 3. Mass $\left(M_{*}\right)$ against radius $\left(R_{*}\right)$ from our analysis using the isochrones Python package. It includes results using our SPC analysis (purple), but also shows results using stellar parameters from Niraula et al. (2017) (red), Teske et al. (2018) (blue), and Rodriguez et al. (2018) (yellow). We used both the MIST (squares) and Dartmouth (circles) model grids. We also use both the Gaia (thick lines) and Hipparcos (thin lines) parallaxes as priors. The black squares, with black error bars, show the mean $M_{*}$ and $R_{*}$. It's clear that the Gaia parallax, which is more precise than the Hipparcos parallax, produces results that are more tightly constrained. For the rest of the analysis presented here, we will use the mean $M_{*}$ and $R_{*}$ determined using the Gaia parallax.

$R_{\star}=0.602_{-0.004}^{+0.005} R_{\odot}$. It's clear that the more precise Gaia parallax produces results that are more tightly constrained than those obtained using the Hipparcos parallax. Consequently, we use the results obtained with the Gaia parallax for the rest of the analysis presented here. Similarly, using the Gaia parallax, the isochrones analysis returns $T_{\text {eff }}=4340_{-53}^{+48},[\mathrm{~m} / \mathrm{H}]=-0.26 \pm 0.09, \log g=4.66_{-0.010}^{+0.015}$ (cgs), and $A_{v}=0.22 \pm 0.11$ for the effective temperature, metallicity, surface gravity, and interstellar reddening, respectively. The $T_{\text {eff }}$ and $\log g$ results are consistent with the results from our SPC analysis, but the metallicity is discrepant at $2 \sigma$. It is, however, consistent with some earlier metallicity estimates (Niraula et al. 2017; Teske et al. 2018). The $T_{\text {eff }}, A_{v}$ and $R_{*}$ results are also consistent with results from Gaia Data Release 2 (Gaia Collaboration et al. 2016, 2018). The isochrone analysis also indicates that the star probably has an age of about $10 \mathrm{Gyr}$, with a lower limit (15.87th percentile) of $5 \mathrm{Gyr}$.

\subsection{Stellar kinematics}

Stars presently near the Sun may come from a wide range of Galactic locations. Therefore, stellar space velocity, as a clue to the origin of a star in the Galaxy, is very important. The accurate Gaia parallax (see Table 1), combined with the proper motions and the stellar radial velocity, make it possible to derive reliable space velocities for GJ 9827. The calculation of the space velocity with respect to the Sun is based on the procedure presented by John-
Table 1. GJ 9827 stellar parameters showing the magnitudes, results from our SPC analysis, and the Hipparcos and Gaia parallaxes used as input to the isochrones Python package for estimating the mass, $M_{*}$, and radius $R_{*}$. We don't show the stellar parameters from Niraula et al. (2017), Teske et al. (2018), and Rodriguez et al. (2018) that were also used in the isochrones analysis (see text for details). Also shown are the metallicity, Effective temperature, $\log g$, and final mass and radius estimates obtained from the isochrones analysis using the Gaia parallax as a prior.

\begin{tabular}{|c|c|c|}
\hline Parameter & Description & Value \\
\hline $\begin{array}{l}\text { Other } \\
\text { identifiers }\end{array}$ & $\begin{array}{l}\text { EPIC } 246389858 \\
\text { HIP } 115752 \\
\text { 2MASS J23270480-0117108 }\end{array}$ & \\
\hline$B$ & APASS Johnson $B$ mag & $11.569 \pm 0.034$ \\
\hline$V$ & APASS Johnson $V$ mag & $10.250 \pm 0.138$ \\
\hline $\begin{array}{l}J \\
K\end{array}$ & $\begin{array}{l}\text { 2MASS } J \text { mag } \\
\text { 2MASS } K \text { mag }\end{array}$ & $\begin{array}{l}7.984 \pm 0.02 \\
7.193 \pm 0.02\end{array}$ \\
\hline $\begin{array}{l}\text { WISE2 } \\
\text { WISE3 } \\
\text { WISE4 }\end{array}$ & $\begin{array}{l}\text { WISE2 mag } \\
W I S E 3 \mathrm{mag} \\
W I S E 4 \mathrm{mag}\end{array}$ & $\begin{array}{l}7.155 \pm 0.02 \\
7.114 \pm 0.017 \\
6.957 \pm 0.107\end{array}$ \\
\hline $\begin{array}{l}v \sin i \\
{[\mathrm{~m} / \mathrm{H}]} \\
T_{\text {eff }} \\
\log g\end{array}$ & $\begin{array}{l}\text { Rotational Velocity (SPC) } \\
\text { Metallicity (SPC) } \\
\text { Effective Temperature (SPC) } \\
\text { Surface Gravity (SPC) }\end{array}$ & $\begin{array}{l}<2 \mathrm{~km} \mathrm{~s}^{-1} \\
-0.5 \pm 0.08 \\
4305 \pm 49 \mathrm{~K} \\
4.72 \pm 0.1(\mathrm{cgs})\end{array}$ \\
\hline $\begin{array}{l}\pi_{H i p} \\
\pi_{G A I A}\end{array}$ & $\begin{array}{l}\text { Hipparcos Parallax (mas) } \\
\text { Gaia Parallax (mas) }\end{array}$ & $\begin{array}{l}32.98 \pm 1.76 \\
33.68 \pm 0.06\end{array}$ \\
\hline $\begin{array}{l}{[\mathrm{m} / \mathrm{H}]} \\
T_{\text {eff }}\end{array}$ & $\begin{array}{l}\text { Metallicity (isochrones) } \\
\text { Effective temperature } \\
\text { (isochrones) }\end{array}$ & $\begin{array}{l}-0.26 \pm 0.09 \\
4340_{-53}^{+40} \mathrm{~K}\end{array}$ \\
\hline $\log g$ & surface gravity (isochrones) & $4.66_{-0.010}^{+0.015}(\mathrm{cgs})$ \\
\hline$M_{*}$ & Mass (isochrones) & $0.606_{-0.014}^{+0.020} \mathrm{M}_{\odot}$ \\
\hline$R_{*}$ & Radius (isochrones) & $0.602_{-0.004}^{+0.005} \mathrm{R}_{\odot}$ \\
\hline
\end{tabular}

son \& Soderblom (1987), corrected for the effect of differential galactic rotation (Scheffler et al. 1987), by adopting a solar Galactocentric distance of $8.5 \mathrm{kpc}$ and a circular velocity of $220 \mathrm{~km} \mathrm{~s}^{-1}$. The correction of space velocity to the Local Standard of Rest is based on a solar motion ${ }^{2}$, $(U, V, W)_{\odot}=(10.0,5.2,7.2) \mathrm{km} \mathrm{s}^{-1}$, as derived from Hipparcos data by Dehnen \& Binney (1998). The peculiar space velocity $S$, given by $S=\left(U^{2}+V^{2}+W^{2}\right)^{1 / 2}$, is quoted with all kinematic data in Table 2 (with the exception of the Gaia parallax which is included in Table 1). GJ 9827, shows kinematic properties typical of the thin disk population. We have calculated the probabilities that the star belongs to a specific population, thick (TD), thin disk (D) or stellar halo $(\mathrm{H})$, following the method used by Bensby et al. (2004). On account of these probabilities, we find for GJ 9827 a thickdisk to thin-disk probability ratio of $T D / D=0.05$, implying

2 In the present work, $\vec{U}$ is defined to be positive in the direction of the Galactic center. 
Table 2. Kinematic data.

\begin{tabular}{cc}
\hline \hline Parameter & GJ 9827 \\
\hline$\mu_{\alpha}[\mathrm{mas} / \mathrm{yr}]^{(1)}$ & $376.02 \pm 0.06$ \\
$\mu_{\beta}[\mathrm{mas} / \mathrm{yr}]^{(1)}$ & $216.07 \pm 0.07$ \\
$U_{L S R}\left[\mathrm{~km} \mathrm{~s}^{-1}\right]^{(2)}$ & $-49.4 \pm 0.4$ \\
$V_{L S R}\left[\mathrm{~km} \mathrm{~s}^{-1}\right]^{(2)}$ & $22.9 \pm 0.9$ \\
$W_{L S R}\left[\mathrm{~km} \mathrm{~s}^{-1}\right]^{(2)}$ & $-18.6 \pm 1.1$ \\
$\left.\mathrm{~S}_{\mathrm{km} \mathrm{s}}^{-1}\right]^{(2)}$ & $57.5 \pm 0.6$ \\
\hline \hline
\end{tabular}

References. (1) Gaia Collaboration et al. 2016, 2018; (2) This work (see text).

that the star is clearly identified as a thin-disk object (typical threshold for assignment to thin disk being TD/D less than 0.1 ).

\section{$4 \quad K 2$ PHOTOMETRY AND LIGHT CURVE ANALYSIS}

After the failure of the second of its four reaction wheels, the Kepler spacecraft was re-purposed for an extended $K 2$ mission to obtain high-precision photometry on a set fields near the ecliptic. GJ 9827 was observed from UT 2016 December 16 until UT 2017 March 04, as part of K2 campaign 12 .

Our data reduction and analysis techniques is very similar to that described in Sections 2.2 and 4.1 of Mayo et al. (2018). We also provide a summary of our methods here. We first applied the method developed by Vanderburg \& Johnson (2014) and Vanderburg et al. (2016a) in order to remove the roll systematics introduced by the periodic thruster firing of the Kepler Space Telescope. Next, we removed lowfrequency variations from the light curve via a basis spline. Then we used the BATMAN transit model (Kreidberg 2015) to simultaneously fit the transits of all three planets, assuming non-interaction and circular orbits. The latter assumption seems reasonable, given that the system is old enough for tidal circularisation to have occured (Barnes 2017), and that systems similar to GJ 9827 do tend to have low eccentricities (Van Eylen \& Albrecht 2015). Additionally, as will be discussed in Section 6, the RV analysis is also consistent with the planets having circular orbits.

The model included four global parameters: baseline flux level, a noise parameter, and two quadratic limb darkening coefficients parameterized according to Kipping (2013). Unlike Mayo et al. (2018) we also impose a stellar density prior of $3.92 \pm 0.014 \mathrm{~g} \mathrm{~cm}^{-3}$, determined using the stellar mass and radius determined in Section 3. When imposing this prior, we also assume that the three planets each have circular orbits.

Additionally, each planet had five parameters: the initial epoch (i.e. time of first transit), the period, the inclination, the ratio of planetary to stellar radius $\left(R_{p} / R_{*}\right)$, and the semi-major axis normalized to the stellar radius $\left(a / R_{*}\right)$. All parameters were given a uniform prior except for each planet's $R_{p} / R_{*}$, for which we assumed a log-uniform prior. We estimated these model transit parameters using emcee (Foreman-Mackey et al. 2013), a Python package which performs Markov chain Monte Carlo (MCMC) simulations with

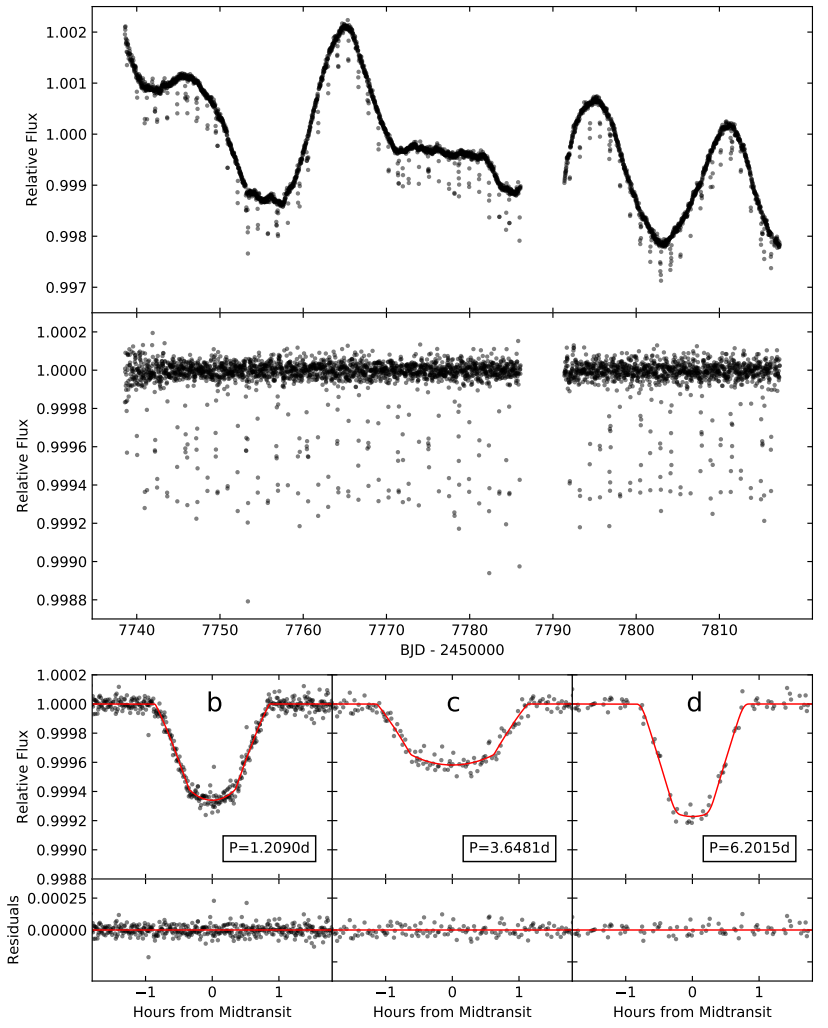

Figure 4. Top panel: $K 2$ lightcurve after removing the roll systematics introduced by the periodic thruster fires of the Kepler Space Telescope, but without the removal of the low-frequency variations. Middle panel: K2 lightcurve after also removing the low-frequency variations. Bottom panel: Phase-folded lightcurves for planets b, c, and d. The model is shown in red and the residuals are shown in the lower parts of the panel.

an affine-invariant ensemble sampler (Goodman \& Weare 2010). Using 38 walkers (i.e. twice the number of model parameters), we ran the MCMC process until convergence, which we defined as the point at which the scale-reduction factor (Gelman \& Rubin 1992) dropped below 1.1 for every parameter.

The systematics corrected, normalised, and phase folded lightcurves are shown in Figure 4. The results of our lightcurve analysis are shown in Table 3. For completeness, the baseline flux level is $1.000 \pm 0.000002$, the noise parameter is $\log ($ jitter $)=-10.11 \pm 0.002$, and the quadratic limb darkening parameters are $q_{1}=0.3999_{-0.1642}^{+0.2403}$ and $q_{2}=0.4372_{-0.2173}^{+0.3004}$. Our results agree well with those in Rodriguez et al. (2018) and Niraula et al. (2017), and suggest that GJ $9827 \mathrm{~b}$ and d, with radii of $R_{p, b}=1.577_{-0.031}^{+0.027}$ and $R_{p, d}=2.022_{-0.043}^{+0.046}$, roughly lie on either side of the radius gap detected by Fulton et al. (2017). The derived quantities in Table $3\left(R_{p}\right.$ and $\left.a\right)$ were determined by sampling the posterior distributions of the dependent quantities, and presenting the median of the resulting distribution with the uncertainties being the difference between this median value and the $16^{\text {th }}$ and $84^{\text {th }}$ percentile values. 
Table 3. Planetary parameters from the light curve analysis.

\begin{tabular}{lllcc}
\hline \hline Parameter & Description & GJ 9827 b & GJ 9827 c & GJ 9827 d \\
\hline$P$ & Period (days) & $1.20898190_{-0.00000714}^{+0.0000093}$ & $3.6480957_{-0.0000621}^{+0.0000633}$ & $6.2014698_{-0.0000611}^{+0.0000626}$ \\
$R_{p} / R_{*}$ & Radius of the planet in stellar radii & $0.02396_{-0.00044}^{+0.00037}$ & $0.01887_{-0.00037}^{+0.00034}$ & $0.03073_{-0.00060}^{+0.00065}$ \\
$R_{p}$ & Radius of the planet (R ( $^{a}$ & $1.577_{-0.031}^{+0.027}$ & $1.241_{-0.026}^{+0.024}$ & $2.022_{-0.043}^{+0.046}$ \\
$T_{C}$ & Time of Transit (BJD-2454833) & $2905.82586_{-0.00026}^{+0.00026}$ & $2909.19930_{-0.00073}^{+0.0072}$ & $2907.96115_{-0.00045}^{+0.00044}$ \\
$T_{14}$ & Transit duration (days) & $0.05270_{-0.00083}^{+0.00093}$ & $0.07604_{-0.00154}^{+0.00154}$ & $0.05095_{-0.00122}^{+0.00147}$ \\
$b$ & Impact parameter & $0.4602_{-0.0443}^{+0.0352}$ & $0.4428_{-0.0483}^{+0.0415}$ & $0.8927_{-0.0090}^{+0.0071}$ \\
$i$ & Inclination & $86.07_{-0.34}^{+0.41}$ & $88.19_{-0.18}^{+0.21}$ & $87.443_{-0.045}^{+0.045}$ \\
$a / R_{*}$ & Semimajor axis in stellar radii & $6.719_{-0.086}^{+0.080}$ & $14.035_{-0.171}^{+0.172}$ & $20.003_{-0.254}^{+0.230}$ \\
$a$ & Semimajor axis (au) & $0.01880_{-0.00014}^{+0.00020}$ & $0.03925_{-0.00029}^{+0.00042}$ & $0.05591_{-0.00041}^{+0.00059}$ \\
\hline
\end{tabular}

Notes. $^{a}$ Radii are derived using our estimate for the stellar radius, $R_{*}=0.602_{-0.004}^{+0.005} R_{\odot}$, and the ratios $R_{\text {planet }} / R_{\text {star }}$ determined here. ${ }^{b}$ Semimajor axes are determined assuming that $M_{\mathrm{S}}+m_{\mathrm{p}} \cong M_{\mathrm{S}}$ and using $a \cong\left[\left(M_{\mathrm{S}} \cdot G\right)^{\frac{1}{3}} \cdot P_{\mathrm{p}}^{\frac{2}{3}}\right] /(2 \pi)^{\frac{2}{3}}$, where $G$ is the gravitational constant.

\section{STELLAR ACTIVITY}

Characterizing the activity level of the host star, and eventually modelling the activity contribution to the RV, is mandatory for accurate mass determination of small planets, even when the star is just moderately active (e.g., Haywood et al. 2018). The $K 2$ light curve shows a strong modulation with peak-to-peak amplitude of $\simeq 0.003$ mag, suggesting a nonnegligible level of activity for this star.

Previous analyses have estimated GJ 9827's rotation period, but the results are not consistent. Niraula et al. (2017) suggests a rotation period of $\sim 17$ days, while Rodriguez et al. (2018) and Teske et al. (2018) suggest a rotation period of 31 days.

Correcting for activity induced signals in the radial velocity data requires an accurate estimate of the star's rotational period. We use the combined HARPS and HARPS-N dataset to carry out a periodogram analysis of the BIS and the FWHM of the CCF, as computed by the DRS (see Section 2.1), and the activity index derived from the Calcium $\mathrm{H}$ and $\mathrm{K}$ lines $\left(\mathrm{S}_{\mathrm{HK}}\right.$, see Table 6 and Tables 2 and 3 in PrietoArranz et al. 2018). Specifically, we used the Bayesian formalism for the generalised Lomb-Scargle periodogram first presented by Mortier et al. (2015). The spectral window of the HARPS and HARPS-N data shows a peak at $\sim 27$ days due to the Moon's sidereal month. This hampers our ability to best exploit our data to derive a reliable measure of the stellar rotational period. When analysing the activity indices, a significant signal is, however, found in the $\mathrm{S}_{\mathrm{HK}}$ data at $\sim 34$ days with another peak at around $\sim 15$ days. We also consider correlations between the activity indices and the RVs. The Spearman's rank correlation coefficients are all below 0.3 .

Therefore, we also carry out a frequency analysis of the combined HARPS and HARPS-N RV data using the Iterative Sine-Wave Fitting (ISWF) method (Vaníček 1971). The power spectra shows clear peaks at $f_{b}=0.827 \mathrm{~d}^{-1}$ (corresponding to the orbital period of GJ $9827 \mathrm{~b}, P_{b}=1.209$ days) and at $f_{d}=0.161 \mathrm{~d}^{-1}$ (corresponding to the orbital period of GJ $9827 \mathrm{~d}, P_{d}=6.21$ days). The low-amplitude signal due to GJ 9827 c can be seen in the power spectrum, but it does not stand out above the noise. This frequency analysis also shows peaks at $f=0.0325 \mathrm{~d}^{-1}, 2 f, 3 f$. The frequency $f$ corresponds to a period of 30.8 days and is clearly related to the stellar rotation period. This would seem to indicate that the $\sim 15$ day signal seen in the $\mathrm{S}_{\mathrm{HK}}$ data is probably the first harmonic of the stellar rotation period.

To better quantify the stellar activity, we carry out an analysis using the $K 2$ light curve (see top panel of Figure 4) but after removing the points affected by transits. We initially determined the auto correlation of the $K 2$ light curve data, computed as described in McQuillan et al. $(2013)^{3}$. This converges to a rotational period of 29 days, which is closer to the 31 days presented in Rodriguez et al. (2018) and Teske et al. (2018), than to the $\sim 17$ days suggested by Niraula et al. (2017).

It has, however, been suggested (Angus et al. 2018) that a Gaussian process (GP) with a quasi-periodic covariance kernel function is a more reliable method to determine the rotational period of active stars. We therefore performed an additional analysis using PyORBIT ${ }^{4}$ (Malavolta et al. 2016), a package for modelling planetary and activity signals. This implements the GP quasi-periodic kernel through the george package (Ambikasaran et al. 2015). For the hyper-parameters we follow the mathematical definition introduced by Grunblatt et al. (2015). Hyper-parameters optimization has been performed using the differential evolution code $\mathrm{pyDE}^{5}$, which provided the starting values for the affine-invariant ensemble sampler emcee (Foreman-Mackey et al. 2013). We followed the same methodology as described in Malavolta et al. (2018).

\footnotetext{
3 As implemented in https://github.com/bmorris3/ interp-acf.

4 Version 5, available at https://github.com/LucaMalavolta/ PyORBIT.

5 Available at https://github.com/hpparvi/PyDE
} 
Table 4. Stellar activity indicators from the GP model using the $K 2$ lightcurve only.

\begin{tabular}{lll}
\hline \hline Parameter & Description & Value \\
\hline$\sigma_{1, \mathrm{jit}, \mathrm{K} 2}{ }^{a}[\mathrm{mag}]$ & Jitter & $0.000006_{-0.000003}^{+0.000003}$ \\
$\sigma_{2, \mathrm{jit}, \mathrm{K} 2^{a}[\mathrm{mag}]}$ & Jitter & $0.000003_{-0.000002}^{+0.000003}$ \\
$\gamma_{1, \mathrm{~K} 2}{ }^{a}[\mathrm{mag}]$ & Offset & $1.000237_{-0.000260}^{+0.000263}$ \\
$\gamma_{2, \mathrm{~K} 2}{ }^{a}[\mathrm{mag}]$ & Offset & $0.999427_{-0.000268}^{+0.000273}$ \\
$P_{\mathrm{rot}}[\mathrm{days}]$ & Rotational period & $28.72_{-0.22}^{+0.18}$ \\
$\lambda[\mathrm{days}]$ & Active region & $33.17_{-6.26}^{+5.90}$ \\
& decay timescale & \\
$w[\mathrm{mag}]$ & Coherence Scale & $0.146_{-0.006}^{+0.006}$ \\
$h_{K 2}$ & Covariance amplitude & $0.00081_{-0.00010}^{+0.00013}$ \\
\hline & & \\
\hline
\end{tabular}

${ }^{a}$ The terms $\sigma_{1, \mathrm{jit}, \mathrm{K} 2}$ and $\gamma_{1, \mathrm{~K} 2}$ are for the K2 data segment ending at BJD-2450000 $=7786.075$, while $\sigma_{2, \mathrm{~K} 2}$ and $h_{2, \mathrm{~K} 2}$ are for the K2 data segment starting at BJD-2450000 $=7791.377$.

Since the GP regression typically scales with the third power of the number of data points, we binned the $K 2$ light curve every 5 points, while ensuring that this did not alter the overall shape and did not change the auto correlation result. Since there is a data gap between BJD - $2450000=7786$ and BJD $-2450000=7791$, we also allow for different offsets and jitters for the two data segments. The GP analysis then suggested a rotational period of $P_{\text {rot }}=28.72_{-0.22}^{+0.18}$ days, a decay timescale of the active regions of $\lambda=33.17_{-6.26}^{+5.90}$ days, and a coherence scale of $w=0.146 \pm 0.006$. We also find a covariance amplitude in the $K 2$ light curve data of $h_{K 2}=0.00081_{-0.00010}^{+0.00013} \mathrm{mag}$. These values are also presented in Table 4.

The GP regression therefore produces a result that is consistent with that from the auto correlation of the $K 2$ light curve data and with that presented in Rodriguez et al. (2018) and Teske et al. (2018). The isochrone analysis also suggests that this star has an age of $\sim 10$ Gyrs, with a lower limit of 5 Gyrs. The stellar kinematics, reported in Section 3.1, indicates that GJ 9827 belongs to the galactic thin disk, but the low metallicity $([\mathrm{m} / \mathrm{H}]=-0.26 \pm 0.09)$ is consistent with this being an older member of that population. A rotation period of $\sim 30$ days is consistent with what would be expected for a star of this age (Reiners \& Mohanty 2012). This would all seem to indicate that the rotation period of GJ 9827 is more likely $\sim 30$ days than the $\sim 17$ days suggested by Niraula et al. (2017). Therefore we will use the results of the GP regression to correct for the stellar activity induced signals in the radial velocity data.

\section{RV ANALYSIS}

The $K 2$ light curve analysis, the ISWF analysis of the HARPS and HARPS-N RVs, and the $\mathrm{S}_{\mathrm{HK}}$ index clearly suggest that the stellar activity of GJ 9827 may have non negligible effects on the RVs. The approach that we've taken is to assume that the light curve variations and activity sig- nals in the RVs can be described by a GP with the same kernel and with common hyper-parameters, except for the covariance amplitude, $h$, which is specific for each dataset. This approach has been quite successful in confirming and improving mass determination of rocky planets (e.g., Haywood et al. 2014, Grunblatt et al. 2015), and it has delivered consistent results with respect to alternative approaches for stellar activity modelling (e.g. Malavolta et al. 2018). In the context of the GP analyis, we take the combined HARPS and HARPS-N RVs to be a single dataset, with the PFS RVs and FIES RVs making up two other datasets. We do, however, allow for an offset between the HARPS and HARPS-N RVs and for independent jitter terms.

We carry out the RV analysis using the PyORBIT code and, as in Section 5, assume that the quasi-periodic kernel is the best choice to model RV variations. When modelling activity signals in RVs with the help of Gaussian processes in a Bayesian framework, imposing priors obtained from the $K 2$ lightcurve on the hyper-parameters of the GP produces statistically indistinguishable results when compared to modelling the RVs and the lightcurve simultaneously (e.g., Malavolta et al. 2018).

Hence, rather than modelling the $K 2$ light curve and the RVs simultaneously, we use the results from Section 5 to set priors on the hyper-parameters, with the exception of the amplitude of the covariance $h$. Since the RV intensity of stellar activity depends on the wavelength range of the instrument and the RV extraction technique (e.g., Zechmeister et al. 2018), for each dataset (combined HARPS and HARPS-N RVs, PFS RVs, and FIES RVs) we used an independent covariance amplitude $h$. For each dataset we also include a jitter term, to compensate for uncorrelated noise not included in the error estimate, and a RV offset. As mentioned above, although we treat the combined HARPS and HARPS-N RVs as a single dataset, we do allow for different offsets and jitter values for the HARPS and HARPS-N RVs. We use uniform priors for both the jitter and the RV offset. We ran two main analyses, one in which the results from Section 5 exactly define the Gaussian priors on the hyperparameters, and one in which they guide our choice of priors, but do not precisely define them. Specifically, in the second analysis we use Gaussian priors on $P_{\text {rot }}, \lambda$, and $w$, with $P_{\text {rot }}=35 \pm 10$ days, $\lambda=36 \pm 15$ days, and $w=0.15 \pm 0.005$.

In the second analysis, we set the $P_{\text {rot }}$ prior to the value we would have used if only spectroscopic activity indexes had been used to estimate the stellar rotation period $(\sim 35$ days from the $\mathrm{S}_{\mathrm{HK}}$ index, see Section 5 ), but we make the range wide enough to also incorporate the results from the analysis using the $K 2$ lightcurve and to account for the photometry and RVs not being simultaneous in time.

In our model we also assume that the orbits of all 3 planets are circular (eccentricity $e=0$ ). In multi-planet systems of close-in planets, the eccentricity evolution depends on both tidal interactions and on eccentricity pumping from planet-planet interactions (Bolmont et al. 2013). However, given the age of the system ( $>5 \mathrm{Gyr}$ ) there has probably been sufficient time for the orbits of these close-in planets to have been tidally circularised (Barnes 2017), and there are indications that systems like GJ 9827 do tend to have low eccentricities (Van Eylen \& Albrecht 2015). We also impose a log-uniform prior on the time of transit centre and a uniform prior on the orbital periods of the 3 planets, taken 
from the results of the analysis discussed in Section 4 (see Table 3).

Our results are shown in Table 5. The table shows the quantities derived from the RVs (radial velocity semiamplitude, $K$, planet mass, $M_{p}$, and mean density, $\rho$ ) and also shows the resulting stellar activity indicators, the uncorrelated jitter, and the RV offset for each dataset. The posterior distributions of some of the fitted parameters from Analysis 1 are shown in Figure 5. For the sake of readability, only the RV semi-amplitude of the planets and the GP hyper parameters are reported. The confidence intervals of the posteriors are computed by taking the $15.87^{\text {th }}$ and $84.14^{\text {th }}$ percentiles of the distribution, except for $K_{\mathrm{c}}$ and $h_{\mathrm{FIES}}$, for which we report the median and the $84.14^{\text {th }}$ percentile.

As discussed above, the two analyses were one in which the activity priors were set by the results of Section 5 , and one in which we used the results of Section 5 to set the region where we'd expect the activity parameters to lie, but set the priors to have a much broader range than that suggested by the results presented in Section 5 . Table 5, shows that the results of these two analyses are consistent. Since the stellar activity indicators derived from the $K 2$ lightcurve, presented in Section 5, probably best represent the stellar activity, we will focus primarily on the results from Analysis 1.

Figure 6 shows the orbital solutions and RV residuals from Analysis 1, for GJ 9827 b (top panel), GJ 9827 c (middle panel), and GJ $9827 \mathrm{~d}$ (lower panel), phased on the period of the corresponding planet and after removing the RV contributions from stellar activity and from the other planets. GJ $9827 \mathrm{~b}$ has a RV semi amplitude of $K_{b}=4.11 \pm 0.40$ $\mathrm{m} \mathrm{s}^{-1}$, suggesting a mass of $M_{p, b}=4.91 \pm 0.49 \mathrm{M}_{\oplus}$. The RV semi amplitude, $K_{c}$, for GJ $9827 \mathrm{c}$ is small and suggests a mass of $M_{p, c}=0.84 \mathrm{M}_{\oplus}$ with an upper limit of $1.50 \mathrm{M}_{\oplus}$, while for GJ $9827 \mathrm{~d}$ the RV semi amplitude is $K_{d}=1.97 \pm 0.40 \mathrm{~m} \mathrm{~s}^{-1}$ with a resulting mass estimate of $M_{p, d}=4.04_{-0.84}^{+0.82} \mathrm{M}_{\oplus}$. The mass estimate for GJ $9827 \mathrm{~b}$ therefore has a precision of better than $10 \%$, while that for GJ $9827 \mathrm{~d}$ is close to $20 \%$.

Figure 7 shows the HARPS-N (filled and open circles), HARPS (open squares), and FIES (red triangles) RVs, together with the best-fit model which includes the planets' signals and the GP model of the correlated stellar noise (light blue curve). Also shown is the GP solution (dashed blue curve) and its associated uncertainty range (grey shaded region).

We don't, however, show the PFS RVs in Figure 7 . What Figure 6 shows is that the RV residuals for some of the PFS data are considerably larger than that for the other datasets. This is most likely because the PFS data covers a long time interval and, during some periods, is insufficiently well sampled to constrain the stellar activity.

To test the consequences of this, we carried out two more analyses, both using the same activity priors as used by Analysis 1 in Table 5. In one we excluded PFS data that appeared to be insufficiently well sampled to constrain the stellar activity, and in the other we used HARPS-N and HARPS data only. In the first of these analyses, we retained the 6 PFS RVs between $\mathrm{BJD}=2455428.80$ and $\mathrm{BJD}=2455439.82$, the $12 \mathrm{PFS} \mathrm{RVs}$ between $\mathrm{BJD}=2455785.72$ and $\mathrm{BJD}=2455485.70$, and the 3 PFS RVs between BJD $=2456139.86$ and $\mathrm{BJD}=2456150.83$.
In both cases, the results were consistent with, and of a similar precision to, those presented in Table 5. Consequently, we conclude that the PFS sampling does not significantly influence our results, both in terms of the best estimate or the precision.

We also carried out one additional analysis, using the same activity priors as in Analysis 1, in which we relax the constraint that the planet eccentricities are all zero. We do, however, constrain the eccentricities to be less than 0.2 , which is based on pure N-body simulations using mercury6 that indicate that this is required for stability. The results from this analysis do allow for the planets to have small eccentricities, but the resulting RV semi-amplitudes, and planet masses, are very close to those produced by the equivalent analysis with circular orbits. The resulting eccentricities are also consistent with $e=0$ at $2.45 \sigma$ which suggests that this result is not significant (Lucy \& Sweeney 1971). There is therefore no strong evidence to indicate that the orbits are non-circular.

\section{DISCUSSION}

Our analysis has allowed us to estimate the masses of GJ $9827 \mathrm{~b}$ and d with a precision of better than $10 \%$ and close to $20 \%$, respectively. We can't, however, put a strong constraint on the mass of GJ 9827 c. Our analysis suggests an upper limit (84\%) for GJ 9827 c's RV semi-amplitude of $<1 \mathrm{~m} \mathrm{~s}^{-1}$. If we assume an Earth-like composition $\left(M_{p} \simeq 1.9 M_{\oplus}\right.$, similar to Kepler-78b, Pepe et al. 2013), the RV semi-amplitude for this planet would be $\simeq 1 \mathrm{~m} \mathrm{~s}^{-1}$. This might suggest that GJ9827c is unlikely to have an Earth-like composition.

Figure 8 shows GJ 9827 b, c, and d on a mass-radius diagram which also includes all planets with a measured mass and radius from the Extrasolar Planets Encyclopae$\mathrm{dia}^{6}$. The data points are shaded according to the precision of their mass estimate and are color-coded according to their incident flux, relative to that of the Earth. The dashed lines show different compositions, taken from Zeng et al. (2016), plus one as yet unpublished track for a planet in which $\mathrm{H}_{2}$ makes up $1 \%$ of its mass. The figure also shows the Earth and Venus, for reference, and indicates the approximate location of the radius gap (Fulton et al. 2017).

GJ 9827 b is consistent with having a rocky, terrestrial (Earth-like) composition. The result for GJ 9827 c suggests that it is not consistent with being rocky, and that water could still make up a substantial fraction of its mass. There are, however, indications that non-detections, like that of GJ 9827 c, could return RV semi-amplitudes that are biased low with respect to the real RV semi-amplitudes (e.g., Damasso et al. 2018). Therefore, our results cannot be interpreted as strong evidence for GJ 9827 c not being rocky. GJ 9827 b, on the other hand, would seem to be composed mostly of silicates and iron. The best estimate suggests that its iron core makes up about $25 \%$ of its mass, similar to that for the Earth and Venus, and the density estimate would seem to rule out GJ $9827 \mathrm{~b}$ having $\mathrm{H} / \mathrm{He}$ on its surface, or the presence of a thick envelope of volatiles.

The bulk density of GJ 9827 d, and its location in the

6 Available at http://www. exoplanet.eu 
Table 5. Best-fit solutions from the RV analyses.

\begin{tabular}{|c|c|c|}
\hline \multirow[t]{2}{*}{ Parameter } & \multicolumn{2}{|c|}{ Best-fit value } \\
\hline & Analysis $1^{a}$ & Analysis $2^{b}$ \\
\hline \multicolumn{3}{|c|}{$\begin{array}{l}\text { Stellar activity GP model. } \\
\text { (Does not include those from the } \\
K 2 \text { analysis presented in Table } 4 \text {.) }\end{array}$} \\
\hline$h_{\text {HARPS }-\mathrm{N}, \text { HARPS }}\left[\mathrm{m} \mathrm{s}^{-1}\right]$ & $2.49_{-0.39}^{+0.48}$ & $2.85_{-0.51}^{+0.66}$ \\
\hline$h_{\mathrm{FIES}}\left[\mathrm{m} \mathrm{s}^{-1}\right]$ & $1.76_{-1.21}^{+2.67}$ & $2.09_{-1.46}^{+3.64}$ \\
\hline$h_{\mathrm{PFS}}\left[\mathrm{ms}^{-1}\right]$ & $3.73_{-1.03}^{+0.93}$ & $3.88_{-0.95}^{+0.95}$ \\
\hline$\lambda[$ days $]$ & $34.77_{-5.64}^{+5.57}$ & $30.97_{-11.79}^{+12.47}$ \\
\hline$w$ & $0.147 \pm 0.006$ & $0.196_{-0.038}^{+0.041}$ \\
\hline$P_{\text {rot }}[$ days $]$ & $28.72_{-0.19}^{+0.19}$ & $30.13_{-2.02}^{+11.11}$ \\
\hline \multicolumn{3}{|l|}{ Uncorrelated jitter } \\
\hline$\sigma_{\mathrm{jit}, \mathrm{HARPS}}\left[\mathrm{ms}^{-1}\right]$ & $0.80_{-0.44}^{+0.42}$ & $0.81_{-0.44}^{+0.41}$ \\
\hline$\sigma_{\mathrm{jit}, \mathrm{FIES}}\left[\mathrm{m} \mathrm{s}^{-1}\right]$ & $1.23_{-0.85}^{+1.58}$ & $1.24_{-0.86}^{+1.65}$ \\
\hline$\sigma_{\mathrm{jit}, \mathrm{PFS}}\left[\mathrm{m} \mathrm{s}^{-1}\right]$ & $2.32_{-1.18}^{+1.28}$ & $2.17_{-1.04}^{+1.17}$ \\
\hline \multicolumn{3}{|l|}{ RV offset } \\
\hline$\gamma_{\text {HARPS }-\mathrm{N}}\left[\mathrm{m} \mathrm{s}^{-1}\right]$ & $31949.335_{-0.753}^{+0.775}$ & $31949.473_{-0.916}^{+0.970}$ \\
\hline$\gamma_{\text {HARPS }}\left[\mathrm{m} \mathrm{s}^{-1}\right]$ & $31948.292_{-0.876}^{+0.907}$ & $31948.556_{-1.027}^{+1.103}$ \\
\hline$\gamma_{\text {FIES }}\left[\mathrm{m} \mathrm{s}^{-1}\right]$ & $31775.640_{-1.988}^{+1.969}$ & $31775.623_{-2.192}^{+2.222}$ \\
\hline$\gamma_{\mathrm{PFS}}\left[\mathrm{ms}^{-1}\right]$ & $0.447_{-0.979}^{+0.984}$ & $0.533_{-0.988}^{+1.019}$ \\
\hline \multicolumn{3}{|c|}{ Quantities derived from RVs } \\
\hline$K_{\mathrm{b}}\left[\mathrm{m} \mathrm{s}^{-1}\right]$ & $4.11_{-0.40}^{+0.40}$ & $4.10_{-0.37}^{+0.37}$ \\
\hline$K_{\mathrm{c}}^{1}\left[\mathrm{~m} \mathrm{~s}^{-1}\right]$ & $0.49(<0.87)$ & $0.39(<0.74)$ \\
\hline$K_{\mathrm{d}}\left[\mathrm{m} \mathrm{s}^{-1}\right]$ & $1.97_{-0.40}^{+0.40}$ & $1.80_{-0.48}^{+0.43}$ \\
\hline$M_{\mathrm{p}, \mathrm{b}}\left(M_{\oplus}\right)$ & $4.91_{-0.49}^{+0.49}$ & $4.90_{-0.45}^{+0.45}$ \\
\hline$M_{\mathrm{p}, \mathrm{c}}{ }^{1}\left(M_{\oplus}\right)$ & $0.84(<1.50)$ & $0.67(<1.27)$ \\
\hline$M_{\mathrm{p}, \mathrm{d}}\left(M_{\oplus}\right)$ & $4.04_{-0.84}^{+0.82}$ & $3.71_{-0.99}^{+0.90}$ \\
\hline$\rho_{\mathrm{b}}^{2}\left[\mathrm{~g} \mathrm{~cm}^{-3}\right]$ & $6.93_{-0.76}^{+0.82}$ & $6.90_{-0.71}^{+0.76}$ \\
\hline$\rho_{\mathrm{c}}^{1,2}\left[\mathrm{~g} \mathrm{~cm}^{-3}\right]$ & $2.42(<4.35)$ & $1.93(<3.66)$ \\
\hline$\rho_{\mathrm{d}}^{2}\left[\mathrm{~g} \mathrm{~cm}^{-3}\right]$ & $2.69_{-0.57}^{+0.58}$ & $2.46_{-0.66}^{+0.63}$ \\
\hline
\end{tabular}

${ }^{a}$ PyORBIT analysis in which we set priors on $P_{\text {rot }}, \lambda$, and $w$ from the stellar activity analysis using the $K 2$ lightcurve only. See Section 5 and Table $4 .{ }^{b}$ PyORBIT analysis in which we use the results of the activity analysis described in 5 to guide our choice of priors, rather than using these results exactly. Specifically, we impose Gaussian priors on $P_{\text {rot }}, \lambda$, and $w$ with $P_{\text {rot }}=35 \pm 10$ days, $\lambda=36 \pm 15$ days, and $w=0.15 \pm 0.05 .{ }^{1}$ For upper limits, we report the median and the $84^{\text {th }}$ percentile. ${ }^{2}$ The density was determined by sampling the posterior distributions for the mass and radius, and presenting the median, $16^{\text {th }}$ and $84^{\text {th }}$ percentiles of the resulting distribution.

mass-radius diagram (Figure 8), suggests that it probably does retain a reasonably substantial atmosphere, with water potentially making up a substantial fraction of its mass. GJ 9827 would therefore appear to host a super-Earth that is probably rocky (GJ $9827 \mathrm{~b}$ ) and one (GJ $9827 \mathrm{~d}$ ) that probably retains a substantial atmosphere. These two planets appear to bracket the radius gap suggested by Fulton et al. (2017) and Van Eylen et al. (2018).

The stellar fluxes received by GJ $9827 \mathrm{~b}$ and c are about 316 and 73 times that received by the Earth, respectively. If they are both rocky, they may still have formed with a com- position similar to that of GJ $9827 \mathrm{~d}$, but may have since lost their atmospheres through photo-evaporation (Lopez \& Fortney 2014). If, however, water still makes up a substantial fraction of GJ 9827 c's mass, then this could have implications for the formation of this system. It could suggest that GJ 9827 c and d both formed beyond the snowline, with GJ $9287 \mathrm{~b}$ forming inside the snowline. Migration could then have produced the configuration we see today. That the system is in a near 1:3:5 resonance (Prieto-Arranz et al. 2018) might be consistent with this scenario. In such a scenario 


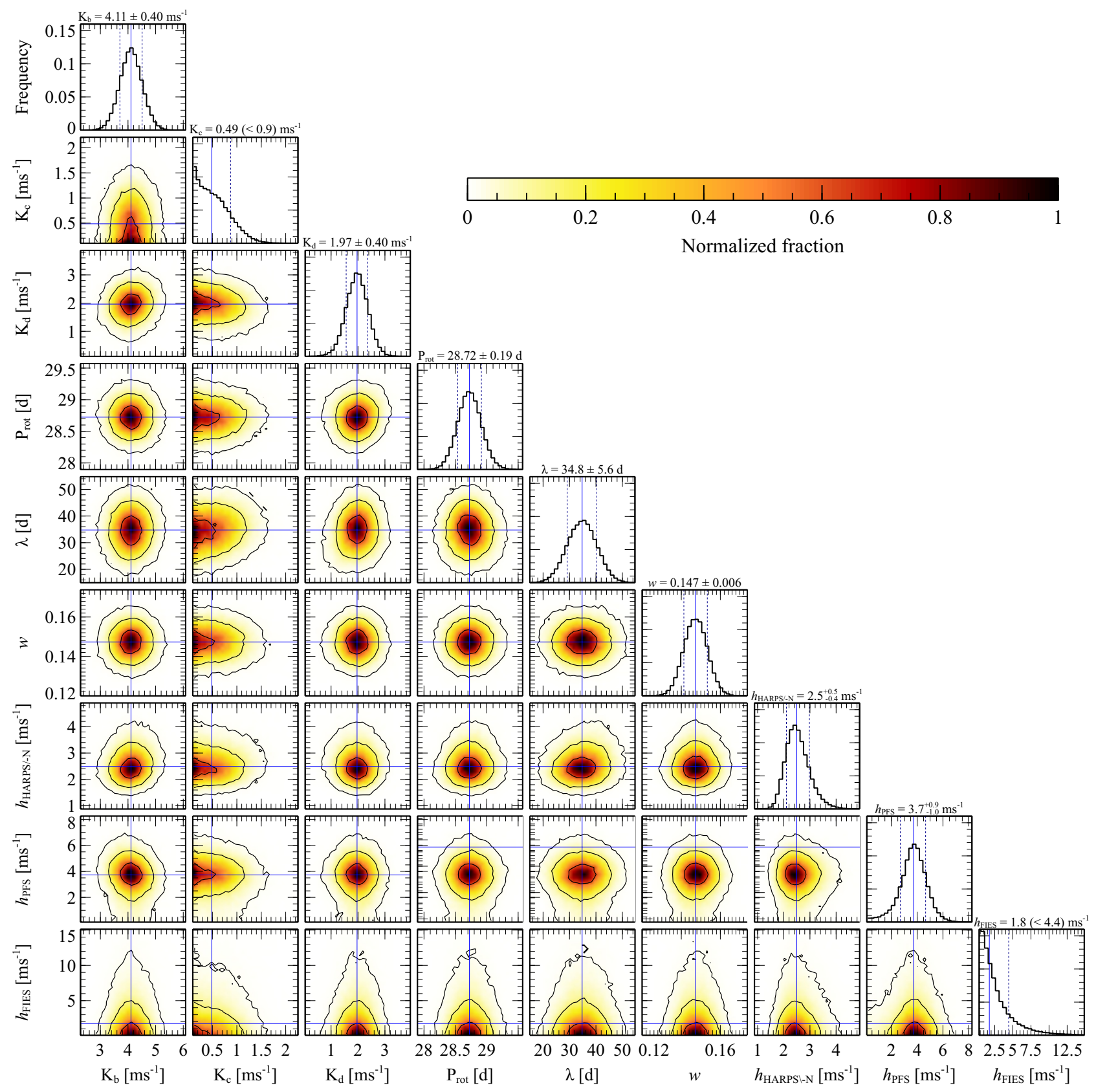

Figure 5. Posterior distributions of some of the fitted parameters determined by the analysis of the GJ 9827 RVs (Analysis 1 in Table $5)$. For the sake of readability, we only show the RV semi-amplitude of the planets and the GP hyper parameters.

GJ 9827 c could still retain a water-rich atmosphere even at its current level of irradiation (Lopez 2017).

On the other hand, the stellar flux received by GJ 9827 $\mathrm{d}$ is about 36 times that received by the Earth, which may not be sufficient for GJ 9827 d to have lost much of its primordial atmosphere (Owen \& Wu 2013; Lopez \& Fortney 2013), whether water-rich or predominantly H/He (Lopez 2017). This system may therefore be consistent with photoevaporation playing a key role in generating the radius gap suggested by Owen \& Wu (2013) and Lopez \& Fortney (2013), and first detected by Fulton et al. (2017).

In fact, if those planets above the radius gap typically retain a $\mathrm{H} / \mathrm{He}$ atmosphere, then a prediction of the photo- evaporation model is that planets just above, and just below, the radius gap should have similar masses, since the envelope should make up only a small fraction of the mass of those just above the gap (Lopez \& Rice 2018). The similar masses of GJ $9827 \mathrm{~b}$ and d are intriguingly consistent with this prediction.

There are, however, alternative explanations. For example, the luminosity of the cooling core could completely erode light envelopes, while having little impact on heavier envelopes (Ginzburg et al. 2018). This would produce a deficit of intermediate-mass planets and, hence, may also explain the observed radius gap (Fulton et al. 2017). Systems 

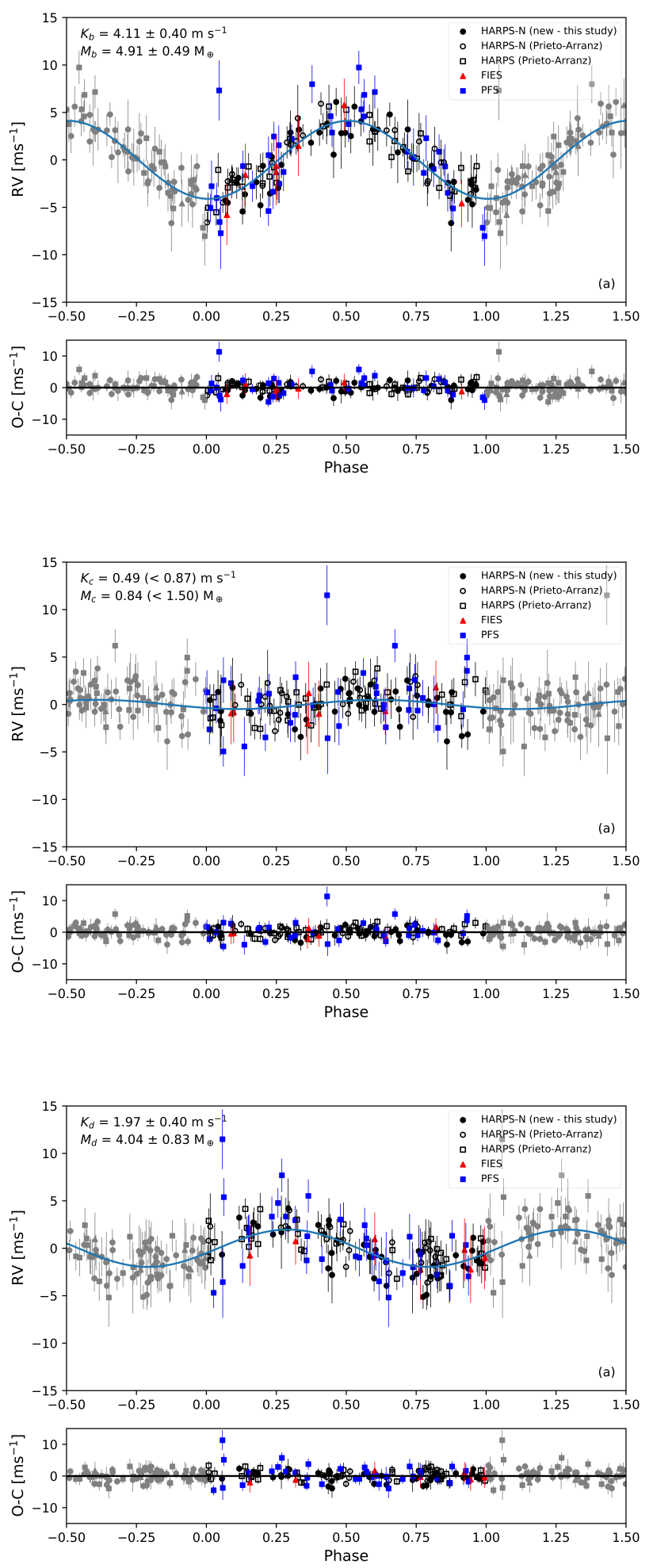

Figure 6. Orbital solutions and RV residuals for GJ 9827 b (top panel), GJ 9827 c (middle panel), and GJ 9827 d (lower panel), phased on the period of the corresponding planet and with the RV contributions from the other planets removed. The details are discussed in Section 6, and these figures show the results from Analysis 1.

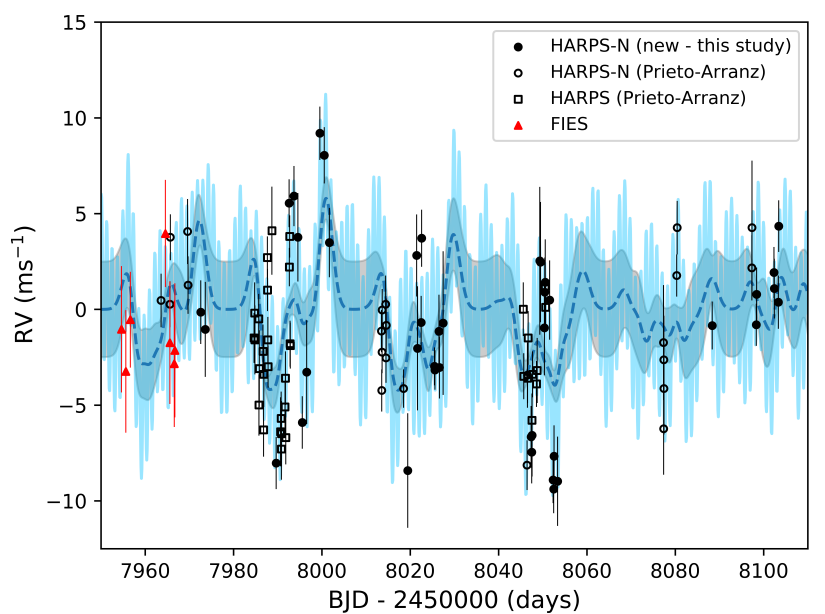

Figure 7. HARPS-N (filled and open circles), HARPS (open squares), and FIES (red triangles) RVs, together with the best-fit models which includes the planets' signals and the GP model of the correlated stellar noise (light blue curve). Also shown is the GP solution (dashed blue curve) and its associated uncertainty range (grey shaded region).

like GJ 9827 will therefore play a key role in determining which of these scenarios most likely explains this radius gap.

\subsection{The composition of planets below the radius gap}

One of the goals of the HARPS-N Collaboration is to try to determine the typical composition of planets with radii similar to that of the Earth. In particular, are planets below the radius gap first clearly mapped by Fulton et al. (2017) rocky? It has already been suggested (Rogers 2015) that most planets above this gap still retain significant envelopes of volatiles, but it's not yet clear if most planets below the gap are primarily composed of silicates and iron.

In Figure 9 we plot the same data as in Figure 8, but scale the planet masses according to the minimum mass they would need, given their radius, in order to be rocky (see composition curves in Figure 8). As in Figure 8, the data points are shaded according to the precision of their mass estimate and are color-coded according to their incident flux, relative to the Earth. We also show the approximate location of the radius gap (Fulton et al. 2017).

What Figure 9 shows quite clearly is that those with radii above the gap, including GJ $9827 \mathrm{~d}$, tend to have masses below that required for them to be rocky, while those below the gap tend to have masses above the mass at which they would be rocky. Our estimate for GJ 9827 b suggests that it is clearly rocky. The upper limit for GJ 9827 c suggests that it is not rocky and that it may still retains a reasonable amount of water, and other volatiles. However, as highlighted in Damasso et al. (2018), there are indications that a result like that for GJ 9827 c could be biased low, so we really can't rule out that GJ 9827 c is indeed rocky.

However, what Figure 9 also shows is that the only other 


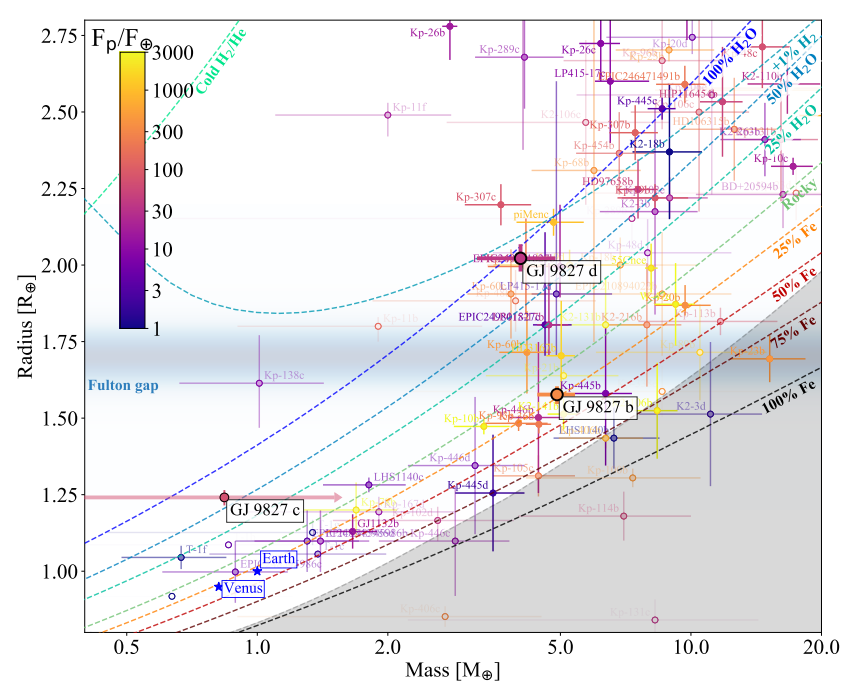

Figure 8. Mass-Radius diagram for GJ 9827 b, GJ 9827 c, and GJ 9827 d together with all planets with a measured mass and radius from the Extrasolar Planets Encyclopaedia. The dashed lines show different compositions, taken from Zeng et al. (2016), plus one additional as yet unpublished track for a planet in which $\mathrm{H}_{2}$ makes up $1 \%$ of its mass. The data points are shaded according to the precision of their mass estimate and are color-coded according to their incident flux. Also shown are Earth and Venus, for reference, and we indicate the approximate location of the radius gap (Fulton et al. 2017).

known planet below the radius gap that is inconsistent with being rocky at $1 \sigma$ is Trappist- $1 \mathrm{f}$. Trappist- $1 \mathrm{f}$ is, however, around a very-low-mass star and has a low bolometric irradiation $\left(M_{*} \sim 0.08 M_{\odot}\right.$ and $F_{p} / F_{\oplus} \sim 0.382$, Gillon et al. 2017). It is quite strongly irradiated in the XUV (Wheatley et al. 2017; Bolmont et al. 2017), but probably does still retain a volatile-rich envelope (Quarles et al. 2017). If GJ 9827 $\mathrm{c}$ does indeed still retain a substantial gaseous envelope, then it would be one of the most heavily irradiated planets below the radius gap to do so, and the only one orbiting an FGK star.

\section{CONCLUSIONS}

Here we present the results of our analysis of the GJ 9827 planetary-system, a system already known to contain 3 super-Earths (Niraula et al. 2017; Rodriguez et al. 2018). We repeat the $K 2$ lightcurve analysis and recover planetary radii that are consistent with these earlier analyses. We then carry out an RV analyses using the Magellan/PFS and FIES radial velocities first presented by Teske et al. (2018) and Niraula et al. (2017) respectively, the HARPS and HARPS$\mathrm{N}$ radial velocities presented by (Prieto-Arranz et al. 2018), and with 41 additional new RV observations from HARPS-N (Cosentino et al. 2012).

Although our RV analysis can't provide a strong constraint on the mass of GJ 9827 c, we can estimate the masses of GJ $9827 \mathrm{~b}$ and d with precisions of better than $10 \%$ (b) and close to $20 \%$ (d). We find that GJ $9827 \mathrm{~b}$ is probably rocky, with an iron core, but is unlikely to have a mass as

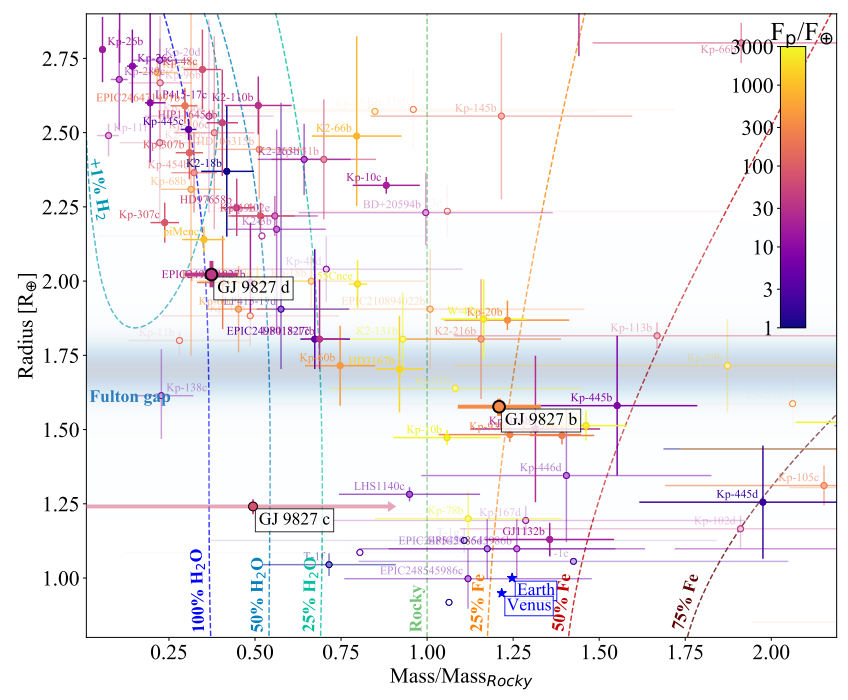

Figure 9. Similar to Figure 8, except the masses are scaled according to the minimum mass they would need, given their radius, to be rocky. As in Figure 8, the data points are shaded according to the precision of their mass estimate and color-coded according to their incident flux, relative to that of the Earth. It also illustrates the location of the radius gap (Fulton et al. 2017).

high as suggested by Teske et al. (2018). GJ 9827 d, on the other hand, almost certainly retains a significant envelope of volatiles.

Using HARPS, HARPS-N and FIES RVs, PrietoArranz et al. (2018) also estimated the masses of the planets in the GJ 9827 system. They conclude that GJ 9827 b is probably rocky, with an iron core, and that GJ $9827 \mathrm{~d}$ still retains an evelope of volatiles, which is consistent with the results presented here. However, our estimates for the mass of GJ 9827 b and GJ 9827 d are inconsistent with their estimates at the $1 \sigma$ level. Our analysis suggests that both GJ $9827 \mathrm{~b}$ and GJ $9827 \mathrm{~d}$ have higher masses than suggested by Prieto-Arranz et al. (2018). Their estimate for GJ 9827 b is still consistent with an Earth-like composition, but their estimate for GJ $9827 \mathrm{~d}$ would seem to suggest a much lower density than is suggested by our analysis.

Prieto-Arranz et al. (2018) also claim a $2 \sigma$ detection for the mass of GJ 9827 c, while we can only really set an upper limit. Although our upper limit is consistent, at $1 \sigma$, with their result, their analysis suggests that GJ 9827 c may well be rocky, whereas ours suggests that it probably is not. It would seem quite important to understand this difference, since the composition of GJ 9827 c could constrain where the planets in this system formed. If water makes up a significant fraction of its mass, then that might suggest that the outer planets in this system formed beyond the snowline. If not, then in situ formation is still a possibility (Chiang \& Laughlin 2013). It is possible, however, that our non-detection has returned RV semi-amplitudes that are biased low (Damasso et al. 2018).

GJ 9827 is particularly interesting system since it hosts a rocky super-Earth near the lower boundary of the radius gap detected by Fulton et al. (2017) and one that retains a substantial atmosphere near the upper boundary of this gap. Consequently, this system could be consistent with 
the inner-most one being sufficiently strongly irradiated to have lost its atmosphere via photo-evaporation (Lopez 2017; Owen \& Wu 2017). If GJ 9827 d retains a low-mass H/He envelope, rather than a water-rich atmosphere, then GJ 9827 $\mathrm{b}$ and $\mathrm{d}$ having similar masses is also consistent with the photoevaporation model. However, we can't yet exclude alternative explanations, such as the luminosity of the cooling core eroding the lighter envelopes (Ginzburg et al. 2016, 2018). Therefore, understanding systems like GJ 9827 will help to determine which scenario is most likely.

Our results also have implications for the typical composition of planets below the radius gap detected by Fulton et al. (2017). Most planets with well-constrained masses below this radius gap have compositions consistent with them being rocky. This is indeed the case for GJ $9827 \mathrm{~b}$, but our analysis can't rule out that GJ 9827 c still retains a waterrich atmosphere. However, if this is the case, GJ $9827 \mathrm{c}$ would be the one of the most heavily irradiated super-Earths below the radius gap that still retains a substantial volatile envelope. Given the faintness of the star $(\mathrm{V}=10.3)$ and the expected RV amplitude ( $1 \mathrm{~m} \mathrm{~s}^{-1}$ for a rocky composition), it seems likely that only the next generation of high-precision velocimeters on large telescopes, such as ESPRESSO (Pepe et al. 2010) or G-CLEF (Szentgyorgyi et al. 2012), will allow a mass determination that has sufficient precision (better than $\sim 20 \%$ ) to uncover its internal composition.

As already highlighted by Rodriguez et al. (2018) and Niraula et al. (2017), GJ 9827 is bright, and cool, and hence is a potential target for atmospheric characterisation via transit spectroscopy (Seager \& Sasselov 2000). The expected signal can be calculated from the planet and star's radii, and the scale-height of the planet's atmosphere (Vanderburg et al. 2016b). Our analysis suggests that if both GJ $9827 \mathrm{~d}$ and GJ $9827 \mathrm{c}$ have predominantly $\mathrm{H} / \mathrm{He}$ envelopes, the atmospheric signal could be as high as a few 100 ppm, which could be detected by the Hubble Space Telescope. However, if their atmospheres are predominantly water, a detection may require the James Webb Space Telescope. That GJ 9827 probably hosts a rocky super-Earth and one that probably retains a substantial atmosphere, and that these two planets bracket the radius gap detected by (Fulton et al. 2017), makes it a particularly interesting target.

\section{ACKNOWLEDGEMENTS}

L.M. and D.M. acknowledge support from INAF/Frontiera through the "Progetti Premiali" funding scheme of the Italian Ministry of Education, University, and Research. Some of this work has been carried out within the framework of the NCCR PlanetS, supported by the Swiss National Science Foundation. A.V. is supported by the NSF Graduate Research Fellowship, grant No. DGE 1144152. This work was performed in part under contract with the California Institute of Technology (Caltech)/Jet Propulsion Laboratory (JPL) funded by NASA through the Sagan Fellowship Program executed by the NASA Exoplanet Science Institute (A.V. and R.D.H.). A.C.C. acknowledges support from STFC consolidated grant number ST/M001296/1. D.W.L. acknowledges partial support from the Kepler mission under NASA Cooperative Agreement NNX13AB58A with the
Smithsonian Astrophysical Observatory. C.A.W. acknowledges support by STFC grant ST/P000312/1. X.D. is grateful to the Society in Science-Branco Weiss Fellowship for its financial support. This material is based upon work supported by the National Aeronautics and Space Administration under grants No. NNX15AC90G and NNX17AB59G issued through the Exoplanets Research Program. This publication was made possible through the support of a grant from the John Templeton Foundation. The opinions expressed are those of the authors and do not necessarily reflect the views of the John Templeton Foundation. The HARPS$\mathrm{N}$ project has been funded by the Prodex Program of the Swiss Space Office (SSO), the Harvard University Origins of Life Initiative (HUOLI), the Scottish Universities Physics Alliance (SUPA), the University of Geneva, the Smithsonian Astrophysical Observatory (SAO), and the Italian National Astrophysical Institute (INAF), the University of St Andrews, Queen's University Belfast, and the University of Edinburgh. This paper includes data collected by the $\mathrm{Ke}$ pler mission. Funding for the Kepler mission is provided by the NASA Science Mission directorate. Some of the data presented in this paper were obtained from the Mikulski Archive for Space Telescopes (MAST). STScI is operated by the Association of Universities for Research in Astronomy, Inc., under NASA contract NAS5-26555. Support for MAST for non-HST data is provided by the NASA Office of Space Science via grant NNX13AC07G and by other grants and contracts. This research has made use of NASA's Astrophysics Data System and the NASA Exoplanet Archive, which is operated by the California Institute of Technology, under contract with the National Aeronautics and Space Administration under the Exoplanet Exploration Program.

\section{REFERENCES}

Ambikasaran S., Foreman-Mackey D., Greengard L., Hogg D. W., O'Neil M., 2015, IEEE Transactions on Pattern Analysis and Machine Intelligence, 38

Angus R., Morton T., Aigrain S., Foreman-Mackey D., Rajpaul V., 2018, MNRAS, 474, 2094

Baranne A., et al., 1996, A\&AS, 119, 373

Barnes R., 2017, Celestial Mechanics and Dynamical Astronomy, 129,509

Batalha N. M., et al., 2013, ApJS, 204, 24

Bensby T., Feltzing S., Lundström I., 2004, A\&A, 415, 155

Bolmont E., Selsis F., Raymond S. N., Leconte J., Hersant F., Maurin A.-S., Pericaud J., 2013, A\&A, 556, A17

Bolmont E., Selsis F., Owen J. E., Ribas I., Raymond S. N., Leconte J., Gillon M., 2017, MNRAS, 464, 3728

Buchhave L. A., et al., 2012, Nature, 486, 375

Buchhave L. A., et al., 2014, Nature, 509, 593

Chen J., Kipping D., 2017, ApJ, 834, 17

Chiang E., Laughlin G., 2013, MNRAS, 431, 3444

Cosentino R., et al., 2012, in Ground-based and Airborne Instrumentation for Astronomy IV. p. $84461 \mathrm{~V}$, doi:10.1117/12.925738

Cosentino R., et al., 2014, in Ground-based and Airborne Instrumentation for Astronomy V. p. 91478C, doi:10.1117/12.2055813

Crane J. D., Shectman S. A., Butler R. P., 2006, in Society of Photo-Optical Instrumentation Engineers (SPIE) Conference Series. p. 626931, doi:10.1117/12.672339

Cutri R. M., et al. 2014, VizieR Online Data Catalog, 2328

Damasso M., et al., 2018, A\&A, 615, A69 
Table 6. HARPS-N radial velocity data.

\begin{tabular}{|c|c|c|c|c|c|c|}
\hline $\begin{array}{l}\text { BJD }_{\text {UtC }} \\
\text { (d) }\end{array}$ & $\begin{array}{l}\mathrm{RV} \\
\left(\mathrm{m} \mathrm{s}^{-1}\right)\end{array}$ & $\begin{array}{l}\sigma_{\mathrm{RV}} \\
\left(\mathrm{m} \mathrm{s}^{-1}\right)\end{array}$ & $\begin{array}{l}\mathrm{BIS}_{\mathrm{span}} \\
\left(\mathrm{m} \mathrm{s}^{-1}\right)\end{array}$ & $\begin{array}{l}\text { FWHM } \\
\left(\mathrm{km} \mathrm{s}^{-1}\right)\end{array}$ & $\begin{array}{l}\mathrm{S}_{\mathrm{HK}} \\
(\mathrm{dex})\end{array}$ & $\begin{array}{l}\sigma_{\mathrm{S}} \mathrm{HK} \\
(\mathrm{dex})\end{array}$ \\
\hline 2457972.581025 & 31949.19 & 1.66 & 54.56 & 6.16146 & 0.760934 & 0.012149 \\
\hline 2457973.598897 & 31948.29 & 2.48 & 47.05 & 6.08261 & 0.703956 & 0.018964 \\
\hline 2457989.650249 & 31941.31 & 1.36 & 44.43 & 6.13164 & 0.690250 & 0.008613 \\
\hline 2457992.585770 & 31954.88 & 1.25 & 47.19 & 6.13195 & 0.712646 & 0.007336 \\
\hline 2457993.670628 & 31955.25 & 1.57 & 52.67 & 6.13304 & 0.734660 & 0.010788 \\
\hline 2457994.574853 & 31953.10 & 2.31 & 44.91 & 6.13740 & 0.716458 & 0.018600 \\
\hline 2457995.576615 & 31943.43 & 1.37 & 47.27 & 6.13503 & 0.738125 & 0.008665 \\
\hline 2457996.564509 & 31946.06 & 2.71 & 62.28 & 6.14124 & 0.703724 & 0.025410 \\
\hline 2457999.535982 & 31958.53 & 1.39 & 46.43 & 6.14647 & 0.744546 & 0.009172 \\
\hline 2458000.538638 & 31957.38 & 1.47 & 50.08 & 6.15661 & 0.758144 & 0.010064 \\
\hline 2458001.680612 & 31952.82 & 1.81 & 43.66 & 6.15685 & 0.754857 & 0.013624 \\
\hline 2458019.448280 & 31940.92 & 2.99 & 43.19 & 6.12897 & 0.680988 & 0.030242 \\
\hline 2458021.448466 & 31952.15 & 2.14 & 50.83 & 6.12614 & 0.684284 & 0.018574 \\
\hline 2458021.635511 & 31947.30 & 3.24 & 55.74 & 6.12959 & 0.709314 & 0.035249 \\
\hline 2458022.469453 & 31948.65 & 1.39 & 44.09 & 6.12319 & 0.674695 & 0.008879 \\
\hline 2458022.578398 & 31953.05 & 1.49 & 41.74 & 6.12904 & 0.692022 & 0.009983 \\
\hline 2458025.479998 & 31946.36 & 0.99 & 49.06 & 6.13471 & 0.700214 & 0.004776 \\
\hline 2458025.580458 & 31946.17 & 1.03 & 48.23 & 6.13419 & 0.709150 & 0.005181 \\
\hline 2458026.500839 & 31948.19 & 1.91 & 52.53 & 6.14859 & 0.722292 & 0.014968 \\
\hline 2458026.617340 & 31946.30 & 1.62 & 57.31 & 6.13966 & 0.715850 & 0.011669 \\
\hline 2458027.436449 & 31948.62 & 3.75 & 49.25 & 6.13452 & 0.761666 & 0.039726 \\
\hline 2458047.362466 & 31942.68 & 1.61 & 51.38 & 6.13719 & 0.734700 & 0.012005 \\
\hline 2458047.488963 & 31941.88 & 1.63 & 48.83 & 6.13660 & 0.718712 & 0.011596 \\
\hline 2458047.572523 & 31942.76 & 2.15 & 55.64 & 6.14504 & 0.719076 & 0.018844 \\
\hline 2458049.334881 & 31951.87 & 3.86 & 55.60 & 6.13803 & 0.680903 & 0.045143 \\
\hline 2458049.484976 & 31951.80 & 3.14 & 54.38 & 6.12018 & 0.682102 & 0.031959 \\
\hline 2458050.373485 & 31948.37 & 2.48 & 51.30 & 6.13536 & 0.642163 & 0.022632 \\
\hline 2458050.447982 & 31950.23 & 1.81 & 45.18 & 6.12398 & 0.686098 & 0.013886 \\
\hline 2458050.564769 & 31950.75 & 2.24 & 47.89 & 6.12488 & 0.636900 & 0.019858 \\
\hline 2458051.552113 & 31949.82 & 2.07 & 42.45 & 6.13584 & 0.675663 & 0.018119 \\
\hline 2458052.333890 & 31940.43 & 1.21 & 47.34 & 6.13214 & 0.665658 & 0.007353 \\
\hline 2458052.475119 & 31939.95 & 1.26 & 51.87 & 6.12258 & 0.650656 & 0.007588 \\
\hline 2458052.551769 & 31941.67 & 1.61 & 50.37 & 6.11954 & 0.680874 & 0.012084 \\
\hline 2458053.372143 & 31940.36 & 2.33 & 41.75 & 6.11748 & 0.669119 & 0.021128 \\
\hline 2458088.371319 & 31948.49 & 1.25 & 52.18 & 6.12414 & 0.693171 & 0.006900 \\
\hline 2458098.320396 & 31948.53 & 1.10 & 49.90 & 6.12255 & 0.702622 & 0.005724 \\
\hline 2458098.426520 & 31950.12 & 1.40 & 45.10 & 6.11918 & 0.712788 & 0.009317 \\
\hline 2458102.331265 & 31951.26 & 1.33 & 45.02 & 6.11895 & 0.704454 & 0.008007 \\
\hline 2458102.409463 & 31950.42 & 1.56 & 50.57 & 6.12284 & 0.696478 & 0.010865 \\
\hline 2458103.342309 & 31949.71 & 1.39 & 54.92 & 6.10729 & 0.749587 & 0.008941 \\
\hline 2458103.418424 & 31953.68 & 1.35 & 49.18 & 6.11461 & 0.737197 & 0.008863 \\
\hline
\end{tabular}

Dehnen W., Binney J. J., 1998, MNRAS, 298, 387

Dotter A., 2016, ApJS, 222, 8

Dotter A., Chaboyer B., Jevremović D., Kostov V., Baron E., Ferguson J. W., 2008, ApJS, 178, 89

Ehrenreich D., et al., 2015, Nature, 522, 459

Feroz F., Hobson M. P., 2008, MNRAS, 384, 449

Feroz F., Hobson M. P., Bridges M., 2009, MNRAS, 398, 1601

Feroz F., Hobson M. P., Cameron E., Pettitt A. N., 2013, preprint, (arXiv:1306.2144)

Foreman-Mackey D., Hogg D. W., Lang D., Goodman J., 2013, PASP, 125, 306

Fulton B. J., Petigura E. A., 2018, preprint, (arXiv:1805.01453)

Fulton B. J., et al., 2017, AJ, 154, 109

Gaia Collaboration et al., 2016, A\&A, 595, A1

Gaia Collaboration Brown A. G. A., Vallenari A., Prusti T., de Bruijne J. H. J., Babusiaux C., Bailer-Jones C. A. L., 2018, preprint, (arXiv:1804.09365)

Gelman A., Rubin D. B., 1992, Statistical Science, 7, 457
Gillon M., et al., 2017, Nature, 542, 456

Ginzburg S., Schlichting H. E., Sari R., 2016, ApJ, 825, 29

Ginzburg S., Schlichting H. E., Sari R., 2018, MNRAS, 476, 759

Goodman J., Weare J., 2010, Communications in Applied Mathematics and Computational Science, Vol. $\sim 5$, No. $\sim 1$, p. $\sim 65-80$, $2010,5,65$

Grunblatt S. K., Howard A. W., Haywood R. D., 2015, ApJ, 808, 127

Haywood R. D., et al., 2014, MNRAS, 443, 2517

Haywood R. D., et al., 2018, AJ, 155, 203

Henden A. A., Levine S., Terrell D., Welch D. L., 2015, in American Astronomical Society Meeting Abstracts \#225. p. 336.16

Houdebine E. R., Mullan D. J., Paletou F., Gebran M., 2016, ApJ, 822, 97

Howard A. W., et al., 2012, ApJS, 201, 15

Inamdar N. K., Schlichting H. E., 2015, MNRAS, 448, 1751

Johnson D. R. H., Soderblom D. R., 1987, AJ, 93, 864

Kipping D. M., 2013, MNRAS, 435, 2152 
Kreidberg L., 2015, PASP, 127, 1161

Lopez E. D., 2017, MNRAS, 472, 245

Lopez E. D., Fortney J. J., 2013, ApJ, 776, 2

Lopez E. D., Fortney J. J., 2014, ApJ, 792, 1

Lopez E. D., Rice K., 2018, MNRAS, 479, 5303

Lopez E. D., Fortney J. J., Miller N., 2012, ApJ, 761, 59

Lucy L. B., Sweeney M. A., 1971, AJ, 76, 544

Malavolta L., et al., 2016, A\&A, 588, A118

Malavolta L., et al., 2018, AJ, 155, 107

Marcus R. A., Sasselov D., Stewart S. T., Hernquist L., 2010, ApJ, 719, L45

Mayo A. W., et al., 2018, AJ, 155, 136

McQuillan A., Aigrain S., Mazeh T., 2013, MNRAS, 432, 1203

Mortier A., Faria J. P., Correia C. M., Santerne A., Santos N. C., 2015, A\&A, 573, A101

Morton T. D., 2015, isochrones: Stellar model grid package, Astrophysics Source Code Library (ascl:1503.010)

Niraula P., et al., 2017, AJ, 154, 266

Owen J. E., Wu Y., 2013, ApJ, 775, 105

Owen J. E., Wu Y., 2017, ApJ, 847, 29

Pepe F., Mayor M., Galland F., Naef D., Queloz D., Santos N. C., Udry S., Burnet M., 2002, A\&A, 388, 632

Pepe F. A., et al., 2010, in Ground-based and Airborne Instrumentation for Astronomy III. p. $77350 \mathrm{~F}$, doi:10.1117/12.857122

Pepe F., et al., 2013, Nature, 503, 377

Prieto-Arranz J., et al., 2018, A\&A, 618, A116

Quarles B., Quintana E. V., Lopez E., Schlieder J. E., Barclay T., 2017, The Astrophysical Journal Letters, 842, L5

Reiners A., Mohanty S., 2012, ApJ, 746, 43

Rodriguez J. E., Vanderburg A., Eastman J. D., Mann A. W., Crossfield I. J. M., Ciardi D. R., Latham D. W., Quinn S. N., 2018, AJ, 155, 72

Rogers L. A., 2015, ApJ, 801, 41

Rogers L. A., Bodenheimer P., Lissauer J. J., Seager S., 2011, ApJ, 738, 59

Scheffler H., Elsässer H., Armstrong A. H., 1987, Physics of the galaxy and interstellar matter

Seager S., Sasselov D. D., 2000, ApJ, 537, 916

Skrutskie M. F., et al., 2006, AJ, 131, 1163

Spada F., Demarque P., Kim Y.-C., Sills A., 2013, ApJ, 776, 87

Szentgyorgyi A., et al., 2012, in Ground-based and Airborne Instrumentation for Astronomy IV. p. $84461 \mathrm{H}$, doi:10.1117/12.924957

Telting J. H., et al., 2014, Astronomische Nachrichten, 335, 41

Teske J. K., Wang S., Wolfgang A., Dai F., Shectman S. A., Butler R. P., Crane J. D., Thompson I. B., 2018, AJ, 155, 148

Van Eylen V., Albrecht S., 2015, ApJ, 808, 126

Van Eylen V., Agentoft C., Lundkvist M. S., Kjeldsen H., Owen J. E., Fulton B. J., Petigura E., Snellen I., 2018, MNRAS, 479,4786

Vanderburg A., Johnson J. A., 2014, PASP, 126, 948

Vanderburg A., et al., 2016a, ApJS, 222, 14

Vanderburg A., et al., 2016b, ApJ, 829, L9

Vaníček P., 1971, Ap\&SS, 12, 10

Weiss L. M., Marcy G. W., 2014, ApJ, 783, L6

Wheatley P. J., Louden T., Bourrier V., Ehrenreich D., Gillon M., 2017, MNRAS, 465, L74

Wolfgang A., Lopez E., 2015, ApJ, 806, 183

Yee S. W., Petigura E. A., von Braun K., 2017, ApJ, 836, 77

Zechmeister M., et al., 2018, A\&A, 609, A12

Zeng L., Sasselov D. D., Jacobsen S. B., 2016, ApJ, 819, 127

Zeng L., et al., 2017, in Lunar and Planetary Science Conference. p. 1576

van Leeuwen F., 2007, A\&A, 474, 653
This paper has been typeset from a $\mathrm{T}_{\mathrm{E}} \mathrm{X} / \mathrm{LAT}_{\mathrm{E}} \mathrm{X}$ file prepared by the author. 